\title{
3 Research Square

\section{Tumor Budding-Derived CCL5 Recruits Fibroblasts to Promote Colorectal Cancer Progression Through CCR5-SLC25A24 Signaling}

\section{Ling-Fang Gao}

Shenzhen Hospital of Southern Medical University

\section{Yan Zhong}

Shenzhen Hospital of Southern Medical University

\section{Ting Long}

Shenzhen Hospital of Southern Medical University

\section{Xia Wang}

Southern Medical University School of Basic Medical Sciences

Jia-Xian Zhu

Shenzhen Hospital of Southern Medical University

Xiao-Yan Wang

Southern Medical University Nanfang Hospital

Zhi-Yan Hu

Southern Medical University Nanfang Hospital

\section{Zuguo Li ( $\nabla$ lizg@smu.edu.cn )}

Shenzhen Hospital of Southern Medical University

\section{Research Article}

Keywords: Tumor budding, CCL5, Fibroblasts, Colorectal cancer, CCR5, SLC25A24, angiogenesis, collagen synthesis

Posted Date: October 19th, 2021

DOI: https://doi.org/10.21203/rs.3.rs-961545/v1

License: (c) (1) This work is licensed under a Creative Commons Attribution 4.0 International License. Read Full License 


\section{Abstract}

Background: Tumor buddings have been included in the routine diagnosis of colorectal cancer (CRC) and considered to be a tumor prognostic factor independent of TNM staging. This study aimed at identifying the contribution of tumor budding-derived $\mathrm{C}-\mathrm{C}$ chemokine ligand 5 (CCL5) to tumor microenvironment (TME) through fibroblasts.

Methods: Co-cultivation recruitment assays and human cytokine array were used to detect the main cytokine derived from CRC tumor buddings to recruit fibroblasts. siRNA transfection and inhibitor treatment were applied to investigate the effective receptor of CCL5 on fibroblasts. Transcriptome sequencing was performed to explore the mechanism inside fibroblasts when stimulated by CCL5. Stimulation with CCL5 in vitro, orthotopic xenograft mouse model and clinical specimens were designed to clarify the contribution of CCL 5 to angiogenesis and collagen synthesis.

Results: H\&E and immunochemistry staining confirmed that CRC with tumor buddings in the invasive front was accompanied by more fibroblasts compared with $\mathrm{CRC}$ without tumor buddings. Further vitro study indicated that CCL5 derived from tumor buddings could recruit fibroblasts through CCR5 receptor on fibroblasts and positively regulate solute carrier family 25 member 24 (SLC25A24) expression in fibroblasts, which could activate pAkt-pmTOR signaling. Moreover, CCL5 can increase the number of a$\mathrm{SMA}^{+} \mathrm{CD} 0^{+} \mathrm{FAP}^{-}$fibroblasts to promote tumor angiogenesis through enhancing the expression of VEGFA and making fibroblasts transdifferentiate into vascular endothelial cells. Meanwhile, CCL5 also can promote collagen synthesis through fibroblasts, thus contributing to tumor progression.

Conclusions: In the invasive front of CRC, tumor-budding-derived CCL5 can recruit fibroblasts through CCR5-SLC25A24 signaling, further promoting angiogenesis and collagen synthesis through fibroblasts, eventually creating a tumor-promoting microenvironment.

\section{Background}

The relationship between tumor and microenvironment is like seed relying on soil. Recent studies have gradually concentrated on TME rather than only tumor itself. Cancer-associated fibroblasts (CAFs), as an indispensable part of tumor stroma, have attracted more attention and been widely concerned by scholars [1-3]. Evidences from clinical and basic researches have shown a strong association between CAFs and poor prognosis in several types of cancer, including breast cancer [4], uterine cervical cancer [5], lung cancer [6], cholangiocarcinoma [7] and CRC [8, 9]. Concretely, the mutually crosstalk between fibroblasts and tumor can activate fibroblasts, which leading to tumor metastasis [8], therapy resistance [10], and immunosuppression [11]. However, the process of how fibroblasts initially become a tumorpromoting phenotype is still unclear.

Tumor budding is defined as a single tumor cell or a cell cluster of up to 4 tumor cells, which is assessed in one hotspot (in a field measuring $0.785 \mathrm{~mm} 2$ ) at the invasive front $[12,13]$. Moreover, there have been studies supporting a close link between tumor budding and a distinctive immune-suppressive 
microenvironment promoting tumor invasion in gastric adenocarcinomas [14], stage I lung adenocarcinoma [15], pancreatic cancer [16] and CRC [17]. As another important component of TME, how fibroblasts crosstalk with tumor buddings remains unknown.

CCL5, also known as Regulated upon Activation, Normal T-cell Expressed, and Secreted (RANTES), interacts with the G-protein-coupled receptors CCR1, CCR3, CCR4, CCR5, GPR75 and CD44 $[18,19]$. It is expressed by $\mathrm{T}$ lymphocytes, macrophages, platelets, synovial fibroblasts, tubular epithelium, and tumor cells [20]. As a chemokine, CCL5 plays an active role in recruiting a variety of leukocytes into inflammatory sites including T cells, macrophages, eosinophils, and basophils. In CRC, Macrophagederived CCL5 facilitates immune escape via the p65/STAT3-CSN5-PD-L1 pathway [21]. Moreover, it has been reported that tumor-derived chemokine CCL5 enhances TGF- $\beta$ secretion of T-regulatory cells through CCL5/CCR5, thereby blocking the killing function of CD8 ${ }^{+} T$ cells [22]. Although CCL5 can promote immunosuppression in CRC, the function of tumor-derived CCL5 to fibroblasts remains elusive.

Here, we first specifically report that CCL5 secreted by CRC tumor buddings in the invasive front can recruit surrounding fibroblasts through CCR5-SLC25A24 pathway, and further promote collagen synthesis and angiogenesis through fibroblasts so as to promote CRC progression. In this context, CCL5 may serve as a potential diagnostic marker and therapeutic target for tumor buddings in CRC.

\section{Methods}

\section{Antibodies, small interfering RNA (siRNA) and primer sequences}

The primary antibodies used in the study are summarized in Additional file 5: Table S1. siRNA and primer sequences used in the study are summarized in Additional file 6: Table S2 and Additional file 7: Table S3. Cell lines and cell culture

The CRC cell lines LS174T (CL-188), RKO (CRL-2577), DLD-1 (CCL-221), Caco2 (HTB-37), SW620 (CCL227), HCT-8 (CCL-244), HCT116 (CCL-247), HCT-15 (CCL-225), normal colorectal epithelia cell line FHC (CRL-1831) and normal colorectal fibroblast cell line CCD-18Co (CRL-1459) were purchased from the American Type Culture Collection (ATCC). All CRC cells were cultured in RPMI 1640 medium (Gibco, USA) supplemented with 10\% fetal bovine serum (FBS) (Gibco, USA). FHC was cultured in RPMI 1640 medium (Gibco, USA) supplemented with 15\% fetal bovine serum (FBS) (Gibco, USA). CCD-18Co was cultured in EMEM medium (ATCC, USA) supplemented with $10 \%$ fetal bovine serum (FBS) (Gibco, USA). They were all cultured at $37^{\circ} \mathrm{C}$ in a humidified atmosphere with $5 \% \mathrm{CO} 2$. Cell line certificates of analysis came from ATCC. All the cell lines were negative for mycoplasma.

\section{Primary normal colorectal fibroblasts extraction and culture}

Fresh normal colorectal mucosae were cut up with surgical scissors and then they were dissociated enzymatically in type IV collagenase $(2.0 \mathrm{mg} / \mathrm{ml}$, Sigma, C5138), hyaluronidase $(0.4 \mathrm{mg} / \mathrm{ml}$, Sigma, 
$\mathrm{H} 1115000)$ and DNase $\left(25 \mathrm{U} / \mathrm{ml}\right.$, Solarbio, D8071) in a $37^{\circ} \mathrm{C}$ constant temperature shaker for $2 \mathrm{~h}$. After $2 \mathrm{~h}$, the tissues were passed through $40-\mu \mathrm{m}$ cell strainer to generate single-cell suspensions. Centrifuge the single cell suspensions at $1200 \mathrm{rpm} / \mathrm{min}$ for $12 \mathrm{~min}$, discard the supernatant, and resuspend the pellet with medium. Primary cells were then plated at a density of $1 \times 105$ viable cells in $25-\mathrm{cm} 2$ adherent flasks in EMEM medium (ATCC, USA) with $10 \%$ FBS (Gibco, USA) in $5 \% \mathrm{CO} 2$ at $37^{\circ} \mathrm{C}$ (trypsin digestion method). The tissues couldn't pass through the strainer were transferred to the $25-\mathrm{cm} 2$ adherent flasks. Add EMEM medium (ATCC, USA) with 10\% FBS (Gibco, USA) into the adherent flasks after 24h when the tissue stuck to the bottom of the adherent flask and waiting for fibroblasts to crawl out of the tissues (improved tissue planting method).

\section{Clinical specimens}

CRC clinical specimens were obtained from patients who were pathologically diagnosed with $C R C$ at Shenzhen Hospital, Southern Medical University. The study was approved by the ethics committee of Shenzhen Hospital, Southern Medical University, China.

\section{Immunohistochemistry (IHC)}

IHC was performed on paraffin-embedded sections of CRC tissue according to standard LSAB protocol (Dako) using primary antibodies against a-SMA, CD90, FAP, CCL5 and CD31. The degree of staining in the sections was observed and scored independently by three pathologists. The percent positivity of CCL5 staining was scored from 0 to 4 : 0 (0-5\%), 1 (6-25\%), 2 (26-50\%), $3(51-75 \%)$, and $4(>75 \%)$. The staining intensity was scored on a 4-point scale: 0 (no staining), 1 (weak staining, light yellow), 2 (moderate staining, yellowish brown), and 3 (strong staining, brown). Subsequently, the CCL5 expression score was calculated through multiplying the percent positivity score and staining intensity score. Accordingly, the expression level of CCL5 was defined as Low (0-4), Medium (5-8), and High (9-12).

\section{Co-culture recruitment assay}

Boyden Transwell chambers (Corning, 353097) were used following the instructions of the manufacturer. Briefly, $2 \times 104$ fibroblasts were incubated into the upper chambers. $1 \times 105$ tumor cells or different concentration of CCL5 cytokine (Peprotech, 300-06-20) were incubated into the lower chamber. After 48h incubation, cells that successfully migrated were fixed with $4 \%$ paraformaldehyde, stained with Hematoxylin and counted in 5 random visual fields using a light microscope.

\section{CCR1 inhibitor or CCR5 inhibitor treatment}

Before the recruitment assay, fibroblasts were pre-cultured with CCR1 inhibitor (BX471, 100nM, MCE, ZK811752) or CCR5 inhibitor (Maraviroc, 100nM, Selleck, UK427857) for 48h. During recruitment assay, $B X 471$ or Maraviroc was put in both of the upper and the lower chambers.

\section{siRNA transfection}

siRNA of CCL5, CCR1, CCR3, CCR4, CCR5, CD44, GPR75 and SLC25A24 were purchased from GenePharma (Shanghai, China). HCT-8, SW620, CCD-18Co and primary normal colorectal fibroblasts were 
transiently transfected with siRNA using Lipofectamine 3000 Transfection Reagent (Invirtrogen, USA) in line with the manufacturer's instructions.

\section{Construction of stable cell lines}

Lentivirus vector carrying the luciferase gene (Luc) and the human CCL5 sequence were obtained from GenePharma (Shanghai, China). An empty vector was used as control to CCL 5 overexpression. According to the manufacturer's instructions, stable cell lines were established by transfection of LS174T and RKO with these lentivirus vectors.

\section{Enzyme linked immunosorbent assay (ELISA)}

CCL5 levels in the conditioned medium (CM) of FHC and CRC tumor cells were measured by ELISA using a commercially available kit (Huamei Biotech, China), as described by the manufacturer's instructions. The results were expressed in $\mathrm{pg} / \mathrm{ml}$, and the standard curve is based on the measured OD value of the standard product.

\section{RNA extraction and quantitative reverse transcription polymerase chain reaction (qRT-PCR)}

Total cellular mRNA was extracted using TRIzol (TaKaRa). Prime-Script RT Reagent Kit with gDNA Eraser (TaKaRa) was used to reverse-transcribed mRNA into cDNA. And SYBR Premix Ex Taq (TaKaRa) was used for qRT-PCR.

\section{Immunoblot}

Total proteins were isolated from cells using RIPA lysis buffer (FDbio, FD008),PMSF (FDbio, FD0100), Protease inhibitors (FDbio, FD1001) and protein phosphatase inhibitors (FDbio, FD1002). The concentration was determined using BCA protein assay kits (FDbio, FD2001). Total proteins were separated by $10 \%$ sodium dodecyl sulfate-polyacrylamide gel electrophoresis (SDS-PAGE) and transferred to polyvinylidene fluoride (PVDF) membrane. The membranes were blocked with $5 \%$ skimmed milk or $5 \% \mathrm{BSA}$ for $1 \mathrm{~h}$ at room temperature and then incubated with primary antibodies at $4^{\circ} \mathrm{C}$ overnight. Subsequently, the membranes were incubated with goat anti-rabbit or anti-mouse secondary antibody (FDbio, FDR007 and FDM007). The protein was detected by ECL chemiluminescence solution (FDbio, FD8030) and visualized using chemiluminescence detection system (Universal Hood II, Bio-Rad). The intensity of the immunoblot bands was quantified with the NIH ImageJ software.

\section{Immunofluorescence of cells and CRC tissue}

Immunofluorescence of cells was performed, as previously described [23]. The steps of immunofluorescence of CRC tissue before incubation of primary antibody were the same as IHC. The following steps after incubation of primary antibody were the same as those of immunofluorescence of cells. Images were captured using the fluorescence inverted microscope.

\section{Orthotopic xenograft colorectal cancer mouse model}


BALB/C-nude mice (male, 3-5 weeks old) were purchased from the Gem Pharmatech Co., Ltd, Guangdong, China. All animal experiments were approved by the ethics committee of Shenzhen Hospital, Southern Medical University, China. 5X106 HCT116 cells were injected subcutaneously into the back of nude mice $(n=3)$. After subcutaneous tumor formation, one of the tumor tissues was fixed and stained with hematoxylin-eosin (H\&E), and the remaining tumor tissues were peeled off and cut into $1 \mathrm{~mm} 3$ tumor tissue pieces with ophthalmic scissors. Choose 5 pieces from the cut tissue pieces and bury them in the cecal serosal layer of nude mice by purse string suture $(n=5)$. After 8 weeks, the ceca of nude mice were surgically removed after euthanasia, fixed in $10 \%$ formalin, embedded in paraffin, and prepared into $2.5 \mu \mathrm{m}$ sections for H\&E staining.

\section{Human cytokine array}

Serum-free CMs from FHC and CRC tumor cells HCT-8, HCT116, HCT-15, SW620 were collected after incubation for $24 \mathrm{~h}$ and filtered through a $0.22-\mu \mathrm{m}$ mesh. The $\mathrm{CM}$ samples were added to antibody arrays against 1000 unique cytokines (RayBio, GSH-CAA-X00) and processed according to the manufacturer's protocol.

\section{Transcriptome sequencing}

Total mRNA was extracted from fibroblasts before and after CCL5 stimulation. Sequencing libraries were generated using NEBNext ${ }^{\circledR}$ UltraTM RNA Library Prep Kit for Illumina ${ }^{\circledR}$ (NEB, USA) following manufacturer's recommendations and index codes were added to attribute sequences to each sample. The clustering of the index-coded samples was performed on a cBot Cluster Generation System using TruSeq PE Cluster Kit v3-cBot-HS (Illumia) according to the manufacturer's instructions.

\section{Sirius Red staining}

Sirius Red staining was performed on paraffin-embedded sections of CRC tissue using Sirius Red Staining Kit (LEAGENE, DC0041), as described by the manufacturer's protocol. The degree of staining in the sections was observed and scored independently by three pathologists under a polarized light microscope. The percent positivity of Sirius Red staining was scored from 0 to 4: 0 (0-5\%), 1 (6-25\%), 2 (26-50\%), $3(51-75 \%)$, and $4(>75 \%)$. The staining intensity was scored on a 4-point scale: 0 (no staining), 1 (weak staining, light orange or green), 2 (moderate staining, medium orange or green), and 3 (strong staining, orange or green). Subsequently, the Sirius Red staining score was calculated through multiplying the percent positivity score and staining intensity score. Accordingly, the level of Sirius Red staining was defined as Low (0-4), Medium (5-8), and High (9-12).

\section{Bioinformatics analysis of relapse-free survival of CRC patients}

The CRC microarray profiles GSE39582 was used to analyze the correlation between the expression of COL1 or COL3 and the relapse-free survival of patients. The chip platform used in this analysis was the Affymetrix Human Genome U133 Plus 2.0 Array. The mRNA values of COL1 or COL3 were divided into 
low and high expression groups according to the median. Thus, each group had mRNA values for 283 cases. Then, the survival curves of the two groups were obtained using the Kaplan-Meier method.

\section{Matrigel angiogenesis experiment}

First, high concentration Matrigel (BD, 354248) was diluted in half. $10 \mu$ l diluted Matrigel was pipetted into each well of $\mu$-Slide Angiogenesis Glass Bottom (Ibidi, 81506) and was allowed to polymerize for 30 minutes at $37{ }^{\circ} \mathrm{C} .1 \times 104$ fibroblasts before or after CCL5 stimulation for $24 \mathrm{~h}$ with $50 \mathrm{ul}$ medium were incubated on high concentration Matrigel. After $2 \mathrm{~h}$, the fibroblasts were fixed with $4 \%$ paraformaldehyde, stained with Hematoxylin and counted in 3 random visual fields using a light microscope. The capillary tubes were quantified by counting the number of lumens.

\section{Statistical analysis}

SPSS software for Mac OS version 25.0 was used for statistical analyses. An unpaired two-tailed Student's t test was used to compare normally distributed data in two groups. Pearson's $\chi 2$ test and Spearman's correlation test were applied to analyze the correlation between the expression of CCL5 or Sirius Red staining and clinicopathological features. Log-rank test was performed in Kaplan-Meier survival curves. The correlated expression levels of CCL 5 and Sirius Red staining in CRC tissue were analyzed by Spearman's correlation test. All data were expressed as the mean \pm standard error of mean (SEM). $\mathrm{P}<0.05$ was considered significant. ns, no significance; $*, P<0.05 ; \star \star, P<0.01 ; * \star \star, P<0.001$.

\section{Results}

\section{CRC tumor cells can recruit fibroblasts around tumor buddings}

Through the clinical observation of 195 cases of CRC tissue with H\&E staining, we found that in the invasive front CRC tissue with tumor buddings was accompanied by more fibroblasts compared with CRC tissue without tumor buddings (Fig. 1a). Moreover, we verified fibroblasts around tumor buddings were highly expressed with a-SMA and CD90, but without the expression of FAP (Fig. 1b). It suggested that tumor buddings in the invasive front of CRC tissue were closely related to the heterogeneity of fibroblasts.

Recently, many studies have revealed that fibroblasts can be recruited by some cytokines secreted by tumor cells or other cells in the TME[7, 24-26]. To study the contribution of CRC tumor cells to fibroblasts, firstly, two types of fibroblasts were used, including normal colorectal fibroblast cell line CCD-18Co and primary normal colorectal fibroblast NF1. Primary normal colorectal fibroblasts were extracted by trypsin digestion or improved tissue planting method (Additional file 1: Figure S1A). To identify these fibroblasts, epithelial marker E-cadherin and fibroblast markers Vimentin and a-SMA were detected by immunofluorescence (Fig. 1c and Additional file 1: Figure S1B).

Secondly, co-culture recruitment assay was conducted, in which CRC tumor cells were incubated in the lower chamber while the fibroblasts were incubated in the upper chamber. The pore size of the co-culture 
upper chamber is $8 \mu \mathrm{m}$, which is enough for fibroblasts to pass through (Fig. 1d). As shown in Fig. 1e, normal colorectal epithelial cell line FHC as well as CRC cell lines LS174T, RKO, DLD-1 and Caco2 had weak recruitment ability towards fibroblasts, while HCT-8, HCT116, HCT-15 and SW620 had stronger recruitment ability towards fibroblasts.

Next, we chose HCT116 for further vivo orthotopic xenograft colorectal cancer mouse experiments, which had stronger recruitment ability towards fibroblasts in vitro. Through the observation of H\&E stained sections of tumors on mice cecum, we found that HCT116 also could recruit fibroblasts in vivo (Fig. 1f). Taken together, these data demonstrated that fibroblasts around tumor buddings could be recruited by CRC tumor cells.

\section{CRC tumor cells in tumor buddings can recruit fibroblasts through CCL5}

To further ascertain the key cytokine secreted by CRC tumor cells for fibroblasts recruitment, the CMs of FHC and 4 CRC tumor cell lines HCT-8, HCT116, HCT-15, SW620, which owned stronger recruitment ability to fibroblast, were detected on a human cytokine array (Fig. 2a). GO function enrichment analysis indicated that differential proteins found above were involved in various activities, including "positive regulation of cell migration"(Fig. 2b) and "extracellular matrix"(Additional file 2: Figure S2A), which suggested that cytokines secreted by CRC tumor cells could recruit other cells in tumor environment and might be correlated with fibroblasts since fibroblasts are the main source of extracellular matrix[1].

Among these proteins, CCL5 was the most up-regulated protein in the CMs of CRC tumor cells compared with FHC (Fig. 1C). Then we verified the mRNA and protein secretion levels of CCL5 in FHC and 8 CRC tumor cells, the results of which were entirely consistent with the initial findings in the co-culture recruitment assay (Fig. 2d, e). Besides, CCD-18Co and primary normal colorectal fibroblasts secreted lower level of CCL5 than FHC (Fig. 2f). Accordingly, it was paracrine not autocrine of CCL5 that recruited fibroblasts. Next, we detected the expression of CCL5 in CRC tissues using IHC and found that CCL5 was highly expressed in tumor buddings among the invasive front (Fig. $2 \mathrm{~g}$ ).

Then, to detect whether CCL5 is the key cytokine in recruiting fibroblasts, we used the co-culture recruitment assay shown as Fig. $2 \mathrm{~h}$. Among different concentrations of CCL5, 40ng/ml CCL5 had the strongest recruitment ability towards fibroblasts (Additional file 2: Figure S2B) and this concentration of CCL5 generally had the ability to recruit CCD-18Co and human colorectal primary fibroblasts (Fig. $2 \mathrm{i}$ and Additional file 2: Figure S2C).

Moreover, to further ascertain whether CCL5 that could recruit fibroblasts was secreted by CRC tumor cells, we constructed CCL5 down-regulating cell lines with siRNA, and constructed CCL5 overexpressing cell lines with lentivirus, and verified the CCL5 mRNA level and protein secretion level for these cell lines (Additional file 2: Figure S2D-G). Then, using the co-culture recruitment assay shown in Fig. 2j, we found that the ability of tumor cells to recruit fibroblasts was significantly weakened after CCL5 interference (Fig. 2k and Additional file 2: Figure S2H), and the same ability was increased after CCL5 overexpression 
(Fig. 2l and Additional file 2: Figure S2I). In this context, the data pointed out that CCL5 was highly expressed in $\mathrm{CRC}$ tumor buddings and can recruit fibroblasts from TME.

\section{CCL5 recruits fibroblasts through CCR5 receptor}

It has been well defined that CCR1, CCR3, CCR4, CCR5, CD44 and GPR75 are probable receptors of CCL5 $[18,19]$. Accordingly, to explore whether CCL5 mediated fibroblasts recruitment through these receptors, firstly, we used siRNA to interfere with them in fibroblasts respectively and verified their interference efficiency (Fig. 3a, b). Next, we conducted the co-culture recruitment assay described as Fig. 3c, and surprisingly found that only downregulation of CCR5 in fibroblasts can significantly reverse the recruitment ability of CCL5 towards fibroblasts (Fig. 3d). Moreover, CCR1 inhibitor BX471 or CCR5 inhibitor Maraviroc were used to treat with fibroblasts respectively for blocking CCR1 or CCR5 before and during co-culture recruitment assay (Fig. 2e). Similarly, results showed that only Maraviroc treatment can significantly weakened the recruitment ability of CCL5 to fibroblasts (Fig. 3f). The above results strongly indicated that CCL5 participated in fibroblasts recruitment through CCR5 receptor.

\section{CCL5 recruits fibroblasts through SLC25A24 inside fibroblasts}

In order to explore the intracellular changes of fibroblasts after CCL5 stimulation, we performed transcriptome sequencing of fibroblasts before and after CCL5 stimulation (Fig. 4a). The top 20 genes were selected and verified in CCD-18Co cell lines and 4 primary fibroblasts with CCL5 stimulation (Additional file 3: Figure S3A), among which SLC25A24 was consistently up-regulated. We subsequently detected that the mRNA level of SLC25A24 was up-regulated in more fibroblasts with CCL5 stimulation (Fig. 4b). SLC25A24,also known as ATP-Mg2/phosphate carrier 1 (APC1), has a regulatory N-terminal domain containing EF-hand $\mathrm{Ca}_{2}{ }^{+}$binding sites which can activate transport during cytosolic $\mathrm{Ca}^{+}$ increases [27-29]. To preliminarily detect the protein expression and location of SLC25A24 in CRC tissue, we conducted immunofluorescence staining and found that SLC25A24 was highly expressed in the fibroblasts surrounding the tumor buddings in the invasive front (Fig. 4c). Then, we selected three fibroblasts to further verify that CCL5 can up-regulate the protein expression of SLC25A24 (Additional file 3: Figure S3B). Functionally, to study whether CCL5 recruited fibroblasts is dependent on SLC25A24, siRNAs of SLC25A25 were used in fibroblasts, and followed by CCL5 recruitment assay (Fig. 4d). Results showed that silencing of SLC25A24 in fibroblasts significantly reduced the recruitment ability of CCL5 (Fig. 4e-g and Additional file 3: Figure S3C). Moreover, immunoblot results demonstrated that the increased expression of SLC25A24 in fibroblasts after CCL5 stimulation relied on CCR5, indicating that CCL5 recruiting fibroblasts was SLC25A24-CCR5 dependent (Additional file 3: Figure S3D).

To further ascertain the pathway activated by CCL5-SLC25A24 in fibroblasts,KEGG signaling pathway enrichment analysis of above transcriptome sequencing was performed which identified that PI3K-Akt signaling pathway was the most relevant after CCL5 stimulation (Fig. 4h). Immunoblot results showed that CCL5 could positively regulate the phosphorylation of Akt and mTOR in fibroblasts (Fig. 4i, left panel). Whereas silencing SLC25A24, phosphorylated Akt and mTOR decreased in fibroblasts even with 
CCL5 stimulation (Fig. 4i, right panel). These findings suggested that CCL5 recruited fibroblasts through SLC25A24-pAkt-pmTOR inside fibroblasts.

\section{CCL5 contributes to the increase of $\mathrm{a}-\mathrm{SMA}^{+} \mathrm{CD}^{-} 0^{+} \mathrm{FAP}^{-}$ fibroblasts to promote tumor angiogenesis}

The above results in Fig. 1b proved that fibroblasts around tumor buddings were specifically expressed with a-SMA ${ }^{+} \mathrm{CD} 90^{+} \mathrm{FAP}^{-}$. To further ascertain whether CCL5 is the main reason accounting for the increase of a-SMA ${ }^{+} \mathrm{CD}^{+} \mathrm{FAP}^{-}$fibroblasts, we performed immunofluorescence and found that the expression of a-SMA and CD90 were increased in fibroblasts with the stimulation of CCL5 (Fig. 5a). VEGFA plays an irreplaceable role in coordinating vascular endothelial cells proliferation/survival, migration and invasion into the surrounding tissue, leading to formation of lumen-containing structures [30] and it has been reported that fibroblasts are the main source of VEGFA other than tumor cells [2]. Furthermore,studies also have shown that fibroblasts can transdifferentiate into vascular endothelial cells to facilitate tumor progression[31-33]. Next, to detect the function of CCL5 on angiogenesis through fibroblasts, immunoblots showed the expression of vascular endothelial markers FLI1, VE-cadherin, CD31 and VEGFA as well as the expression of a-SMA and CD90 were increased in fibroblasts stimulated with CCL5 (Fig. 5b). Functionally, we further found that the angiogenesis ability of fibroblasts was enhanced with CCL5 stimulation (Fig. 5c). Clinically, IHC staining was performed on serial sections from the same CRC tissue with 17 patients in total to detect the expression of CCL5, a-SMA, CD90, FAP and CD31, and found that in the invasive front, CCL5 was highly expressed in the tumor buddings, which were surrounded by accumulated a-SMA ${ }^{+} \mathrm{CD} 90^{+} \mathrm{FAP}^{-}$fibroblasts as well as increased angiogenesis (Fig. $5 \mathrm{~d}$ ). In this context, we pointed out that tumor buddings secreted CCL5 to increase the number of a-SMA $\mathrm{CD}^{+} \mathrm{FAP}^{-}$fibroblasts to promote tumor angiogenesis through increasing the expression of VEGFA and letting fibroblasts transdifferentiate into vascular endothelial cells.

\section{CCL5 promotes collagen synthesis through fibroblasts contributing to tumor progression}

GO function enrichment analysis performed on above transcriptome sequencing indicated that CCL5 was also relevant to enrichment of extracellular matrix (Fig. 6a). It is well known that collagen type I (COL1) and collagen type III (COL3) are the main components of extracellular matrix and they are mainly synthesized and secreted by fibroblasts [2]. To clarify the function of CCL5 on collagen synthesis, we performed immunoblots which demonstrated that CCL5 could increase protein expression of COL1 and COL3 in fibroblasts in vitro (Fig. 6b).

Clinically, to further investigate the role of CCL5 plays in CRC, we detected the expression of CCL5 protein in 195 paraffin-embedded CRC tissues and 162 adjacent normal colorectal tissues. Results showed that CCL5 was significantly higher expressed in CRC tissues than normal tissues (Fig. 6c and Additional file 4: Figure S4A). The correlation between CCL5 expression level and CRC clinical features was analyzed (Table 1), and further Spearman's correlation test showed that high expression of CCL5 was positively 
associated with high risk of the deep tumor invasion ( $r=0.244$, Fig. $6 \mathrm{~d})$, lymph node metastasis $(r=0.237$, Additional file 4: Figure S4C), the presence of peri-intestinal cancer nodules ( $r=0.198$, Additional file 4: Figure S4D) and advanced TNM stages ( $r=0.256$, Additional file 4: Figure S4E). Moreover, to detect collagen distribution in 88 of the above 195 CRC tissues, Sirius Red staining of COL1 (reddish) and COL3 (greenish) was applied. As shown in Fig. 6e and Additional file 4: Figure S4B, the expression of COL1 and COL3 were increased in CRC tissue. The correlation between Sirius Red staining and CRC clinical features was also analyzed (Table 2), and further Spearman's correlation test showed that its high distribution surrounding CRC tumor cells was also positively related to deep tumor invasion ( $r=0.431$, Fig. $6 f$ ), lymph node metastasis ( $r=0.351$, Additional file 4: Figure S4F), the presence of peri-intestinal cancer nodules $(r=0.288$, Additional file 4: Figure S4G) and advanced TNM stages ( $r=0.442$, Additional file 4: Figure S4H). Intriguingly, we observed that high expression of CCL5 in tumor buddings in the invasive front was often accompanied by increased synthesis of collagen (Fig. 6g). Additionally, Spearman's correlation analyses revealed positive correlation between the expression levels of CCL5 in tumor cells and the collagen in surrounding area in serial sections from the same CRC tissue with 88 patients in total $(r=0.317$, Fig. $6 \mathrm{~h}$ ). In vivo, we used HCT116, the CCL5 highly secreted tumor cell line, to establish orthotopic xenograft CRC mouse model and found that collagen synthesis was increased around the tumor cells in the invasive front (Fig. 6i). Furthermore, we used the CRC microarray profiles GSE39582 to analyze the relapse-free survival of COL1 and COL3 and found that higher expression of COL1 and COL3 tightly related to the poorer prognosis of CRC patients (Fig. 6j). Taken together, these data suggested that tumor buddings secreted CCL5 to promote collagen synthesis through fibroblasts, thus contributing to tumor progression.

\section{Discussion}

TME is widely implicated in tumorigenesis because it harbors cancer cells that interacts with surrounding cells to foster the evolution of cancer [34,35]. Inside this microenvironment, fibroblasts have a strong tumor-modulating effect closely related to poor prognosis and recurrence of patients [36-39]. Despite their vital roles in CRC carcinogenesis, there is lack of specific markers to define the heterogeneous of highly complicated fibroblasts populations. In our research, we found that CRC tumor buddings could highly secret CCL5, which could recruit fibroblasts through CCR5-SLC25A24 signaling and form a characteristic fibroblasts cluster with a-SMA ${ }^{+} \mathrm{CD} 90^{+} \mathrm{FAP}^{-}$expression located around the tumor buddings in the invasive front, furthermore facilitate tumor angiogenesis and collagen synthesis (Fig. 6k).

Tumor cells are heterogeneous, and tumor buddings consist of the most aggressive subgroups of tumor cells, which play a leading role in the process of tumor invasion $[12,13]$. However, there is no unique marker for tumor buddings, and it is unreliable to count them only by means of H\&E staining when tumor cells are difficult to distinguish from reactive mesenchymal cells. In our research, CCL 5 was found as a marker for tumor buddings which provides a concretely new evidence for the role of tumor budding in tumor progression. Consequently, the pathological diagnosis of tumor buddings in CRC can be more accurately diagnosed with the expression of CCL5. In addition, studies have shown that the high expression of CCL5 in colorectal cancer tumor cells can promote their proliferation [40]. The high 
secretion of CCL5 in colorectal cancer can also promote the apoptosis of CD8 ${ }^{+} \mathrm{T}$ cells through Tregulatory cells, thereby promoting tumor progression through immunosuppression [22]. Our research makes the role of CCL5 in the progression of CRC more comprehensive and also makes the network of tumor-microenvironment interaction in CRC clearer. Taken together, therapies targeting CCL5 may play a significant role in blocking the progression of colorectal cancer.

The heterogeneity of fibroblasts is also worth of attention. We have found a subgroup of fibroblasts induced by CCL5 with the biomarkers of a-SMA ${ }^{+} \mathrm{CD} 90^{+} \mathrm{FAP}^{-}$, which is mainly located around the tumor buddings. It contributes to multiple tumor-promoting effects, including tumor angiogenesis and collagen synthesis, which provides a new mechanism for tumor budding in tumor progression. It has been found in literature that the circulating tumor cells were detected in the circulation system together with fibroblasts [41-43]. It appears that the infiltration and metastasis of tumor-fibroblast clusters has a greater effect on the prognosis of tumor patients, and the tumor progression might be inhibited by blocking invasive tumor cells together with surrounding fibroblasts. And our research provides another strong evidence for the existence of congregated infiltration and metastasis of tumor cells and fibroblasts. In our research, tumor buddings can recruit fibroblasts through CCR5 receptor in the process of invasion and it is a vital step for further angiogenesis and collagen synthesis. CCR5 inhibitor Maraviroc, as a therapeutic drug for HIV, has been relatively mature in clinical treatment [44-46]. There is also study demonstrated that tumoral immune cells can be targeted effectively in CRC metastasis by anti-CCR5 therapy in cancer patients [47]. Consequently, CCR5 inhibitor can not only target immune cells but also fibroblasts in the invasive front in CRC, suggesting that it is very suitable for the treatment of CRC. Despite all these, further researches are also needed.

The SLC25 carrier family comprises 53 members, most members of which transport solutes across the inner mitochondrial membrane as part of a variety of distinct metabolic processes [48]. SLC25A24 belongs to a subgroup of short calcium-binding mitochondrial carriers (SCaMCs) with four paralogs in mammals: SCaMC-3/SLC25A23, SCaMC-1/SLC25A24, SCaMC-2/SLC25A25, and SCaMC-3like/SLC25A41 [49-51]. These carriers consist of a C-terminal domain containing six transmembrane helices homologous to the mitochondrial carrier proteins and an $\mathrm{N}$-terminal domain with $\mathrm{Ca2}^{+}$-binding $\mathrm{EF}$ hands conferring $\mathrm{Ca}_{2}{ }^{+}$sensitivity to the carrier. As a mitochondrial inner membrane protein, SLC25A24 has been reported to be involved in the uptake and accumulation of adenine nucleotides [52]. In addition, SLC25A24 has also been found to be related to anti-oxidative stress in previous studies [53]. In this study, we found that SLC25A24 was highly expressed in fibroblasts in the invasive front which surrounded tumor buddings. The impact of these SLC25A24 fibroblasts on tumor cells in turn is still unclear and remains to be explored.

\section{Conclusions}

In conclusion, our study suggested that in the invasive front of CRC, tumor-budding-derived CCL5 can recruit fibroblasts through CCR5-SLC25A24 signaling, further promote angiogenesis and collagen 
synthesis through fibroblasts, eventually creating a tumor-promoting microenvironment.

\section{Abbreviations}

CRC: colorectal cancer

CCL5: C-C chemokine ligand 5

SLC25A24: solute carrier family 25 member 24

TME: tumor microenvironment

CAFs: cancer-associated fibroblasts

RANTES: Activation, Normal T-cell Expressed, and Secreted

siRNA: small interfering RNA

FBS: fetal bovine serum

IHC: Immunohistochemistry

ELISA: enzyme linked immunosorbent assay

qRT-PCR: quantitative reverse transcription polymerase chain reaction

CM: conditioned medium

SDS-PAGE: sodium dodecyl sulfate-polyacrylamide gel electrophoresis

PVDF: polyvinylidene fluoride

H\&E: hematoxylin-eosin

COL1: collagen type I

COL3: collagen type III

APC1: ATP-Mg2 ${ }^{+} /$phosphate carrier 1

SCaMCs: short calcium-binding mitochondrial carriers

\section{Declarations}

\section{Ethics approval and consent to participate}

This study was approved by the Ethics Committee of Southern Medical University, GuangZhou, China. 


\section{Consent for publication}

All authors have consented to publication of the results presented in this manuscript.

\section{Availability of data and materials}

All data presented or analyzed in this study are included either in this article or in the additional files.

\section{Competing interests}

The authors have no conflicts of interest to declare.

\section{Funding}

This study was funded by the National Natural Science Foundation of China (No. 81974441).

\section{Authors' contributions}

Zu-Guo Li, Xiao-Yan Wang and Zhi-Yan Hu conceived and designed the study. Ling-Fang Gao, Xia Wang and Jia-Xian Zhu performed the experiments. Ling-Fang Gao interpreted and analyzed the data. LingFang Gao and Yan Zhong wrote and reviewed the manuscript. Ling-Fang Gao and Ting Long prepared figures. All authors read and approved the final manuscript.

\section{Acknowledgments}

Not applicable.

\section{References}

1. Kalluri R. The biology and function of fibroblasts in cancer. Nat Rev Cancer. 2016;16:582-98.

2. Kalluri R, Zeisberg M. Fibroblasts in cancer. Nat Rev Cancer. 2006;6:392-401.

3. Kuzet SE, Gaggioli C. Fibroblast activation in cancer: when seed fertilizes soil. Cell Tissue Res. 2016;365:607-19.

4. Su S, Chen J, Yao H, Liu J, Yu S, Lao L, Wang M, Luo M, Xing Y, Chen F, et al. CD10(+)GPR77(+) Cancer-Associated Fibroblasts Promote Cancer Formation and Chemoresistance by Sustaining Cancer Stemness. Cell. 2018;172:841-56 e816.

5. Murata T, Mizushima H, Chinen I, Moribe H, Yagi S, Hoffman RM, Kimura T, Yoshino K, Ueda Y, Enomoto T, et al. HB-EGF and PDGF mediate reciprocal interactions of carcinoma cells with cancerassociated fibroblasts to support progression of uterine cervical cancers. Cancer Res. 2011;71:663342.

6. Chen WJ, Ho CC, Chang YL, Chen HY, Lin CA, Ling TY, Yu SL, Yuan SS, Chen YJ, Lin CY, et al. Cancerassociated fibroblasts regulate the plasticity of lung cancer stemness via paracrine signalling. Nat Commun. 2014;5:3472. 
7. Cadamuro M, Nardo G, Indraccolo S, Dall'olmo L, Sambado L, Moserle L, Franceschet I, Colledan M, Massani M, Stecca T, et al. Platelet-derived growth factor-D and Rho GTPases regulate recruitment of cancer-associated fibroblasts in cholangiocarcinoma. Hepatology. 2013;58:1042-53.

8. Heichler C, Scheibe K, Schmied A, Geppert Cl, Schmid B, Wirtz S, Thoma OM, Kramer V, Waldner MJ, Buttner C, et al. STAT3 activation through IL-6/IL-11 in cancer-associated fibroblasts promotes colorectal tumour development and correlates with poor prognosis. Gut. 2020;69:1269-82.

9. Hu JL, Wang W, Lan XL, Zeng ZC, Liang YS, Yan YR, Song FY, Wang FF, Zhu XH, Liao WJ, et al. CAFs secreted exosomes promote metastasis and chemotherapy resistance by enhancing cell stemness and epithelial-mesenchymal transition in colorectal cancer. Molecular Cancer. 2019;18:91.

10. Huelsken J, Hanahan D. A Subset of Cancer-Associated Fibroblasts Determines Therapy Resistance. Cell. 2018;172:643-4.

11. Elyada E, Bolisetty M, Laise P, Flynn WF, Courtois ET, Burkhart RA, Teinor JA, Belleau P, Biffi G, Lucito MS, et al. Cross-Species Single-Cell Analysis of Pancreatic Ductal Adenocarcinoma Reveals AntigenPresenting Cancer-Associated Fibroblasts. Cancer Discov. 2019;9:1102-23.

12. Dawson H, Galuppini F, Trager P, Berger MD, Studer P, Brugger L, Zlobec I, Inderbitzin D, Lugli A. Validation of the International Tumor Budding Consensus Conference 2016 recommendations on tumor budding in stage I-IV colorectal cancer. Hum Pathol. 2019;85:145-151.

13. Lugli A, Kirsch R, Ajioka Y, Bosman F, Cathomas G, Dawson H, El Zimaity H, Flejou JF, Hansen TP, Hartmann $A$, et al. Recommendations for reporting tumor budding in colorectal cancer based on the International Tumor Budding Consensus Conference (ITBCC) 2016. Mod Pathol. 2017;30:1299-1311.

14. Zhang N, Wang D, Duan Y, Ayarick VA, Cao M, Wang Y, Zhang G, Wang Y. The special immune microenvironment of tumor budding and its impact on prognosis in gastric adenocarcinoma. Pathol Res Pract. 2020;216:152926.

15. Kadota K, Yeh YC, Villena-Vargas J, Cherkassky L, Drill EN, Sima CS, Jones DR, Travis WD, Adusumilli PS. Tumor Budding Correlates With the Protumor Immune Microenvironment and Is an Independent Prognostic Factor for Recurrence of Stage I Lung Adenocarcinoma. Chest. 2015;148:711-21.

16. Wartenberg M, Cibin S, Zlobec I, Vassella E, Eppenberger-Castori S, Terracciano L, Eichmann MD, Worni M, Gloor B, Perren A, et al. Integrated Genomic and Immunophenotypic Classification of Pancreatic Cancer Reveals Three Distinct Subtypes with Prognostic/Predictive Significance. Clin Cancer Res. 2018;24:4444-54.

17. Guil-Luna S, Mena R, Navarrete-Sirvent C, Lopez-Sanchez LM, Khouadri K, Toledano-Fonseca M, Mantrana A, Guler I, Villar C, Diaz C, et al. Association of Tumor Budding With Immune Evasion Pathways in Primary Colorectal Cancer and Patient-Derived Xenografts. Front Med (Lausanne). 2020;7:264.

18. Dedoni S, Campbell LA, Harvey BK, Avdoshina V, Mocchetti I. The orphan G-protein-coupled receptor 75 signaling is activated by the chemokine CCL5. J Neurochem. 2018;146:526-39.

19. Aldinucci D, Colombatti A. The inflammatory chemokine CCL5 and cancer progression. Mediators Inflamm. 2014;2014:292376. 
20. Aldinucci D, Borghese C, Casagrande N. The CCL5/CCR5 Axis in Cancer Progression. Cancers. 2020;12:1765.

21. Liu C, Yao ZY, Wang JN, Zhang W, Yang Y, Zhang Y, Qu XL, Zhu YB, Zou JJ, Peng SS, et al. Macrophage-derived CCL5 facilitates immune escape of colorectal cancer cells via the p65/STAT3CSN5-PD-L1 pathway. Cell Death Differ. 2020;27:1765-81.

22. Chang LY, Lin YC, Mahalingam J, Huang CT, Chen TW, Kang CW, Peng HM, Chu YY, Chiang JM, Dutta A, et al. Tumor-Derived Chemokine CCL5 Enhances TGF-beta-Mediated Killing of CD8(+) T Cells in Colon Cancer by T-Regulatory Cells. Can Res. 2012;72:1092-102.

23. Hu Z, Long T, Ma Y, Zhu J, Gao L, Zhong Y, Wang X, Wang X, Li Z. Correction to: Downregulation of GLYR1 contributes to microsatellite instability colorectal cancer by targeting p21 via the P38MAPK and PI3K/AKT pathways. J Exp Clin Cancer Res. 2020;39:125.

24. De Vincenzo A, Belli S, Franco P, Telesca M, laccarino I, Botti G, Carriero MV, Ranson M, Stoppelli MP. Paracrine recruitment and activation of fibroblasts by c-Myc expressing breast epithelial cells through the IGFs/IGF-1R axis. Int J Cancer. 2019;145:2827-39.

25. Sasaki S, Baba T, Shinagawa K, Matsushima K, Mukaida N. Crucial involvement of the CCL3-CCR5 axis-mediated fibroblast accumulation in colitis-associated carcinogenesis in mice. Int $\mathrm{J}$ Cancer. 2014;135:1297-306.

26. Tanabe Y, Sasaki S, Mukaida N, Baba T. Blockade of the chemokine receptor, CCR5, reduces the growth of orthotopically injected colon cancer cells via limiting cancer-associated fibroblast accumulation. Oncotarget. 2016;7:48335-45.

27. Fiermonte G, De Leonardis F, Todisco S, Palmieri L, Lasorsa FM, Palmieri F. Identification of the human mitochondrial ATP-Mg/Pi transporter. Bba-Bioenergetics. 2004;1658:191-1.

28. Harborne SPD, Ruprecht JJ, Kunji ERS. Calcium-induced conformational changes in the regulatory domain of the human mitochondrial ATP-Mg/Pi carrier. Bba-Bioenergetics. 2015;1847:1245-53.

29. Monne M, Daddabbo L, Giannossa LC, Nicolardi MC, Palmieri L, Miniero DV, Mangone A, Palmieri F. Mitochondrial ATP-Mg/phosphate carriers transport divalent inorganic cations in complex with ATP. J Bioenerg Biomembr. 2017;49:369-80.

30. Claesson-Welsh L, Welsh M. VEGFA and tumour angiogenesis. J Intern Med. 2013;273:114-27.

31. Frangogiannis NG. Fact and Fiction About Fibroblast to Endothelium Conversion Semantics and Substance of Cellular Identity. Circulation. 2020;142:1663-6.

32. Han JK, Chang SH, Cho HJ, Choi SB, Ahn HS, Lee J, Jeong H, Youn SW, Lee HJ, Kwon YW, et al. Direct conversion of adult skin fibroblasts to endothelial cells by defined factors. Circulation. 2014;130:1168-78.

33. Kurian L, Sancho-Martinez I, Nivet E, Aguirre A, Moon K, Pendaries C, Volle-Challier C, Bono F, Herbert $\mathrm{JM}$, Pulecio J, et al. Conversion of human fibroblasts to angioblast-like progenitor cells. Nat Methods. 2013;10:77-83.

34. Hanahan D, Weinberg RA. The hallmarks of cancer. Cell. 2000;100:57-70. 
35. Hanahan D, Weinberg RA. Hallmarks of cancer: the next generation. Cell. 2011;144:646-74.

36. Isella C, Terrasi A, Bellomo SE, Petti C, Galatola G, Muratore A, Mellano A, Senetta R, Cassenti A, Sonetto $C$, et al. Stromal contribution to the colorectal cancer transcriptome. Nat Genet. 2015;47:312-9.

37. Kasashima H, Duran A, Martinez-Ordonez A, Nakanishi Y, Kinoshita H, Linares JF, Reina-Campos M, Kudo Y, L'Hermitte A, Yashiro M, et al. Stromal SOX2 Upregulation Promotes Tumorigenesis through the Generation of a SFRP1/2-Expressing Cancer-Associated Fibroblast Population. Dev Cell. 2021;56:95.

38. Wang W, Tang YA, Xiao Q, Lee WC, Cheng B, Niu Z, Oguz G, Feng M, Lee PL, Li B, et al. Stromal induction of BRD4 phosphorylation Results in Chromatin Remodeling and BET inhibitor Resistance in Colorectal Cancer. Nat Commun. 2021;12:4441.

39. Yu M, Guo G, Huang L, Deng L, Chang CS, Achyut BR, Canning M, Xu N, Arbab AS, Bollag RJ, et al. CD73 on cancer-associated fibroblasts enhanced by the A2B-mediated feedforward circuit enforces an immune checkpoint. Nat Commun. 2020;11:515.

40. Niu MK, Yi M, Dong B, Luo SX, Wu KM. Upregulation of STAT1-CCL5 axis is a biomarker of colon cancer and promotes the proliferation of colon cancer cells. Annals of Translational Medicine. 2020;8:951.

41. Ao Z, Shah SH, Machlin LM, Parajuli R, Miller PC, Rawal S, Williams AJ, Cote RJ, Lippman ME, Datar $\mathrm{RH}$, et al. Identification of Cancer-Associated Fibroblasts in Circulating Blood from Patients with Metastatic Breast Cancer. Cancer Res. 2015;75:4681-7.

42. Duda DG, Duyverman AMMJ, Kohno M, Snuderl M, Steller EJA, Fukumura D, Jain RK. Malignant cells facilitate lung metastasis by bringing their own soil. P Natl Acad Sci USA. 2010;107:21677-82.

43. Jones ML, Siddiqui J, Pienta KJ, Getzenberg RH. Circulating fibroblast-like cells in men with metastatic prostate cancer. Prostate. 2013;73:176-81.

44. Gulick RM, Wilkin TJ, Chen YQ, Landovitz RJ, Amico KR, Young AM, Richardson P, Marzinke MA, Hendrix CW, Eshleman SH, et al. Safety and Tolerability of Maraviroc-Containing Regimens to Prevent HIV Infection in Women: A Phase 2 Randomized Trial. Ann Intern Med. 2017;167:384-93.

45. Levy Y, Lelievre JD, Assoumou L, Aznar E, Pulido F, Tambussi G, Crespo M, Meybeck A, Molina JM, Delaugerre C, et al. Addition of Maraviroc Versus Placebo to Standard Antiretroviral Therapy for Initial Treatment of Advanced HIV Infection: A Randomized Trial. Ann Intern Med. 2020;172:297305.

46. Tan Q, Zhu Y, Li J, Chen Z, Han GW, Kufareva I, Li T, Ma L, Fenalti G, Li J, et al. Structure of the CCR5 chemokine receptor-HIV entry inhibitor maraviroc complex. Science. 2013;341:1387-90.

47. Halama N, Zoernig I, Berthel A, Kahlert C, Klupp F, Suarez-Carmona M, Suetterlin T, Brand K, Krauss J, Lasitschka F, et al. Tumoral Immune Cell Exploitation in Colorectal Cancer Metastases Can Be Targeted Effectively by Anti-CCR5 Therapy in Cancer Patients. Cancer Cell. 2016;29:587-601.

48. Cunningham CN, Rutter J. 20,000 picometers under the OMM: diving into the vastness of mitochondrial metabolite transport. EMBO Rep. 2020;21:e50071. 
49. del Arco A, Satrustegui J. Identification of a novel human subfamily of mitochondrial carriers with calcium-binding domains. J Biol Chem. 2004;279:24701-13.

50. Fiermonte G, De Leonardis F, Todisco S, Palmieri L, Lasorsa FM, Palmieri F. Identification of the mitochondrial ATP-Mg/Pi transporter. Bacterial expression, reconstitution, functional characterization, and tissue distribution. J Biol Chem. 2004;279:30722-30.

51. Traba J, Satrustegui J, del Arco A. Characterization of SCaMC-3-like/slc25a41, a novel calciumindependent mitochondrial ATP-Mg/Pi carrier. Biochem J. 2009;418:125-33.

52. Aprille JR. Regulation of the mitochondrial adenine nucleotide pool size in liver: mechanism and metabolic role. FASEB J. 1988;2:2547-56.

53. Traba J, Del Arco A, Duchen MR, Szabadkai G, Satrustegui J. SCaMC-1 promotes cancer cell survival by desensitizing mitochondrial permeability transition via ATP/ADP-mediated matrix $\mathrm{Ca}(2+)$ buffering. Cell Death Differ. 2012;19:650-60.

\section{Tables}


Table 1. The relationship between CCL5 expression and CRC clinicopathological features

\begin{tabular}{|c|c|c|c|c|c|c|}
\hline & $\begin{array}{l}\text { No. of } \\
\text { cases }\end{array}$ & Low & Medium & High & $x^{2}$ value & $\begin{array}{l}P \\
\text { value }\end{array}$ \\
\hline Frequency & $\begin{array}{l}195 \\
\square 100 \% \bigotimes\end{array}$ & $\begin{array}{l}65 \\
\text { \33.3\%】 }\end{array}$ & $\begin{array}{l}92 \\
\square 47.2 \% \bigotimes\end{array}$ & $\begin{array}{l}38 \\
\text { ه19.5\%区 }\end{array}$ & & \\
\hline
\end{tabular}

\section{Age}

\begin{tabular}{|c|c|c|c|c|c|c|}
\hline$<60$ years & $96 \rrbracket 49.2 \% \rrbracket$ & $\begin{array}{l}33 \\
\rrbracket 34.4 \% \rrbracket\end{array}$ & $\begin{array}{l}40 \\
₫ 41.7 \% \rrbracket\end{array}$ & $\begin{array}{l}23 \\
\rrbracket 24.0 \% \rrbracket\end{array}$ & 3.219 & 0.200 \\
\hline$>=60$ years & $99 \rrbracket 50.8 \% \rrbracket$ & $\begin{array}{l}32 \\
\rrbracket 32.3 \% \rrbracket\end{array}$ & $\begin{array}{l}52 \\
₫ 52.5 \% \rrbracket\end{array}$ & 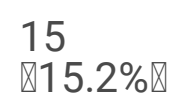 & & \\
\hline
\end{tabular}

\section{Gender}

\begin{tabular}{|c|c|c|c|c|c|c|}
\hline Male & $\begin{array}{l}119 \\
\square 61.0 \% \square\end{array}$ & $\begin{array}{l}43 \\
\rrbracket 36.1 \% \rrbracket\end{array}$ & $\begin{array}{l}50 \\
\varangle 42.0 \% \rrbracket\end{array}$ & $\begin{array}{l}26 \\
\rrbracket 21.8 \% \rrbracket\end{array}$ & 3.317 & 0.190 \\
\hline Female & 76囚39.0\%区 & $\begin{array}{l}22 \\
\rrbracket 28.9 \% \bigotimes\end{array}$ & $\begin{array}{l}42 \\
₫ 55.3 \% \rrbracket\end{array}$ & 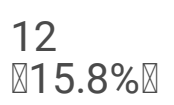 & & \\
\hline
\end{tabular}

\section{Position}

\begin{tabular}{|c|c|c|c|c|c|c|}
\hline Colon & 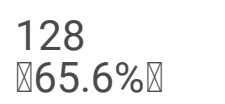 & $\begin{array}{l}41 \\
\rrbracket 32.0 \% \rrbracket\end{array}$ & $\begin{array}{l}61 \\
₫ 47.7 \% \bigotimes\end{array}$ & $\begin{array}{l}26 \\
\text { \20.3\%区 }\end{array}$ & 2.471 & 0.650 \\
\hline Rectum & $58 ه 29.7 \% \bigotimes$ & $20 \otimes 34.5 \rrbracket$ & $\begin{array}{l}26 \\
\varangle 44.8 \% \bigotimes\end{array}$ & 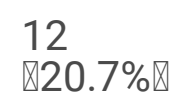 & & \\
\hline Colorectum & $9 \rrbracket 4.6 \% \rrbracket$ & $4 \rrbracket 44.4 \% \bigotimes$ & $\begin{array}{l}5 \\
\text { ه55.6\%区 }\end{array}$ & $0 \rrbracket 0.0 \% \rrbracket$ & & \\
\hline
\end{tabular}

\section{Tumor sizelmaximum} diameter[

\begin{tabular}{|c|c|c|c|c|c|c|}
\hline$<5 \mathrm{~cm}$ & $99 \llbracket 51.0 \% \rrbracket$ & $\begin{array}{l}30 \\
\rrbracket 30.3 \% \rrbracket\end{array}$ & $\begin{array}{l}52 \\
\bigotimes 52.5 \% \rrbracket\end{array}$ & $\begin{array}{l}17 \\
\square 17.2 \% \rrbracket\end{array}$ & 2.155 & 0.340 \\
\hline$>=5 \mathrm{~cm}$ & $95 \rrbracket 49.0 \% \rrbracket$ & $\begin{array}{l}34 \\
\text { 冈35.8\%囚 }\end{array}$ & $\begin{array}{l}40 \\
\bigotimes 42.1 \% \bigotimes\end{array}$ & $\begin{array}{l}21 \\
\rrbracket 22.1 \% \bigotimes\end{array}$ & & \\
\hline
\end{tabular}

\section{Histology}

\begin{tabular}{|c|c|c|c|c|c|c|}
\hline Poor & $22 \rrbracket 11.3 \% \square$ & $6 \rrbracket 27.3 \% \rrbracket$ & $\begin{array}{l}13 \\
\square 59.1 \% \bigotimes\end{array}$ & $\begin{array}{l}3 \\
\square 13.6 \% \bigotimes\end{array}$ & 1.505 & 0.826 \\
\hline Moderate & $\begin{array}{l}155 \\
₫ 79.5 \% \rrbracket\end{array}$ & $\begin{array}{l}53 \\
\rrbracket 34.2 \% \square\end{array}$ & $\begin{array}{l}71 \\
₫ 45.8 \% \bigotimes\end{array}$ & $\begin{array}{l}31 \\
\rrbracket 20.0 \% \rrbracket\end{array}$ & & \\
\hline Well & $18 \otimes 9.2 \% \bigotimes$ & $6 \rrbracket 33.3 \% \rrbracket$ & $\begin{array}{l}8 \\
\triangle 44.4 \% \bigotimes\end{array}$ & 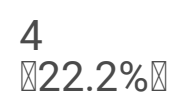 & & \\
\hline
\end{tabular}

\section{T stage}


Tis

$2 \varangle 1.0 \% \rrbracket$

2 $100.0 \% \rrbracket$

$0 \otimes 0.0 \% \bigotimes \quad 0 \otimes 0.0 \% \rrbracket$

22.460

0.004

$\mathrm{T} 1$

$4 \llbracket 2.1 \% \rrbracket$

$3 \otimes 75.0 \% \rrbracket$

1 \25.0\%区

T2

$21 \rrbracket 10.8 \% \rrbracket$

$6 \rrbracket 28.6 \% \rrbracket$

11

\52.4\%区 \19.0\%区

T3

123

$₫ 63.1 \% \rrbracket$

47

$45 \llbracket 23.1 \% \rrbracket$

\38.2\%区

59

$\llbracket 48.0 \% \bigotimes$

17

T4

45囚23.1\%区 7囚15.6\%区

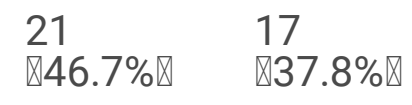

\section{Lymphatic metastasis}

Negative

107

$\triangle 54.9 \% \rrbracket$

46

$\triangle 43.0 \% \rrbracket$

46

$\triangle 43.0 \% \rrbracket$

15

$\triangle 14.0 \% \rrbracket$

$11.154 \quad 0.004$

Positive

$88 \rrbracket 45.1 \% \bigotimes$

19

\21.6\%区

46

$\triangle 52.3 \% \rrbracket$

23

\26.1\%区

\section{Tumor deposits}

Absent

171

\87.7\%】

61

$\llbracket 35.7 \% \rrbracket$

82

$\llbracket 48.0 \% \rrbracket$

28

\16.4\%区

9.366

0.009

Present

$24 \rrbracket 12.3 \% \rrbracket \quad 4 \bigotimes 16.7 \% \rrbracket$

10

10

$\triangle 41.7 \% \square \quad \square 41.7 \% \rrbracket$

\section{Mucinous component}

\begin{tabular}{|c|c|c|c|c|c|}
\hline Absent & $\begin{array}{l}162 \\
\square 83.1 \% \bigotimes\end{array}$ & $\begin{array}{l}53 \\
\varangle 32.7 \% \square\end{array}$ & $\begin{array}{l}77 \\
\varangle 47.5 \% \bigotimes\end{array}$ & 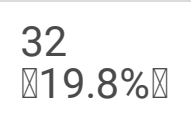 & 0.169 \\
\hline Present & $33 \rrbracket 16.9 \% \rrbracket$ & 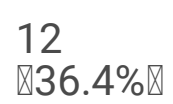 & $\begin{array}{l}15 \\
₫ 45.5 \% \bigotimes\end{array}$ & $\begin{array}{l}6 \\
\rrbracket 18.2 \% \rrbracket\end{array}$ & \\
\hline
\end{tabular}

\section{Distant metastasis}

Negative

182

$\varangle 93.3 \% \square$

63

\34.6\%区

83

$₫ 45.6 \% \rrbracket$

36

$\triangle 19.8 \% \rrbracket$

Positive

$13 \otimes 6.7 \% \rrbracket \quad 2 \bigotimes 15.4 \% \rrbracket$

9

$\llbracket 69.2 \% \square \quad \square 15.4 \% \square$

Microstatellite instability

\begin{tabular}{|c|c|c|c|c|c|c|}
\hline MSS & $\begin{array}{l}161 \\
\otimes 82.6 \% \bigotimes\end{array}$ & $\begin{array}{l}53 \\
\rrbracket 32.9 \% \rrbracket\end{array}$ & $\begin{array}{l}74 \\
\llbracket 46.0 \% \rrbracket\end{array}$ & 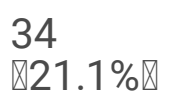 & 4.754 & 0.314 \\
\hline MSI-L & $11 \otimes 5.6 \% \bigotimes$ & $6 \rrbracket 54.5 \% \rrbracket$ & $\begin{array}{l}5 \\
\varangle 45.5 \% \bigotimes\end{array}$ & $0 \rrbracket 0.0 \% \rrbracket$ & & \\
\hline MSI-H & $23 \otimes 11.8 \% \square$ & $6 \rrbracket 26.1 \% \rrbracket$ & $\begin{array}{l}13 \\
₫ 56.5 \% \rrbracket\end{array}$ & $\begin{array}{l}4 \\
\text { ه17.4\%区 }\end{array}$ & & \\
\hline
\end{tabular}




\begin{tabular}{|c|c|c|c|c|c|c|}
\hline Negative & $\begin{array}{l}186 \\
\varangle 95.4 \% \rrbracket\end{array}$ & 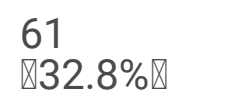 & $\begin{array}{l}90 \\
\mathbb{8} 48.4 \% \bigotimes\end{array}$ & $\begin{array}{l}35 \\
\text { ه18.8\%】 }\end{array}$ & 2.523 & 0.283 \\
\hline Positive & $9 \llbracket 4.6 \% \rrbracket$ & $4 \llbracket 44.4 \% \rrbracket$ & $\stackrel{2}{\triangle 22.2 \% \bigotimes}$ & $\begin{array}{l}3 \\
\text { \33.3\%】 }\end{array}$ & & \\
\hline \multicolumn{7}{|l|}{ TNM stage } \\
\hline 0 & $2 \bigotimes 1.0 \% \bigotimes$ & $2(100.0 \%)$ & $0 \bigotimes 0.0 \% \bigotimes$ & $0 \rrbracket 0.0 \% \rrbracket$ & 41.890 & 0.000 \\
\hline I & $18 \otimes 9.2 \% \rrbracket$ & $8 \rrbracket 44.4 \% \bigotimes$ & $\begin{array}{l}7 \\
\rrbracket 38.9 \% \rrbracket\end{array}$ & $\begin{array}{l}3 \\
\text { ه16.7\%】 }\end{array}$ & & \\
\hline$\| \mathrm{A}$ & $12 \rrbracket 6.2 \% \rrbracket$ & 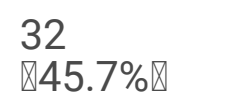 & 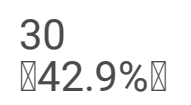 & $\begin{array}{l}8 \\
\square 11.4 \% \bigotimes\end{array}$ & & \\
\hline IIB & $70 \varangle 35.9 \% \rrbracket$ & $2 \rrbracket 22.2 \% \bigotimes$ & $\begin{array}{l}4 \\
\bigotimes 44.4 \% \bigotimes\end{array}$ & $\begin{array}{l}3 \\
\bigotimes 33.3 \% \rrbracket\end{array}$ & & \\
\hline IIIA & $4 \rrbracket 2.1 \% \rrbracket$ & $1 \rrbracket 25.0 \% \bigotimes$ & $\begin{array}{l}2 \\
\square 50.0 \% \bigotimes\end{array}$ & $\begin{array}{l}1 \\
\otimes 25.0 \% \bigotimes\end{array}$ & & \\
\hline IIIB & $56 \rrbracket 28.7 \% \rrbracket$ & $\begin{array}{l}15 \\
\otimes 26.8 \% \bigotimes\end{array}$ & $\begin{array}{l}34 \\
₫ 60.7 \% \rrbracket\end{array}$ & $\begin{array}{l}7 \\
\otimes 12.5 \% \bigotimes\end{array}$ & & \\
\hline IIIC & $24 \rrbracket 12.3 \% \rrbracket$ & $3 \otimes 12.5 \% \bigotimes$ & $\begin{array}{l}7 \\
\square 29.2 \% \rrbracket\end{array}$ & $\begin{array}{l}14 \\
\square 58.3 \% \bigotimes\end{array}$ & & \\
\hline IV & $12 \rrbracket 6.2 \% \rrbracket$ & $2 \rrbracket 16.7 \% \bigotimes$ & $\begin{array}{l}8 \\
\square 66.7 \% \square\end{array}$ & $\begin{array}{l}2 \\
\square 16.7 \% \bigotimes\end{array}$ & & \\
\hline
\end{tabular}

Table 2. The relationship between Sirius Red staining and CRC clinicopathological features 
No. of cases

Frequency

Age

$<60$ years

$>=60$ years
88ख100\%】

38

$\llbracket 43.2 \% \llbracket$
Medium High

28

$\llbracket 31.8 \% \rrbracket$ $x^{2}$ value $\quad \boldsymbol{P}$

value

\section{Gender}

Male

Female

\begin{tabular}{|c|c|c|c|}
\hline $40 \rrbracket 45.5 \% \rrbracket$ & 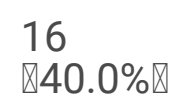 & $\begin{array}{l}12 \\
\rrbracket 30.0 \% \bigotimes\end{array}$ & $\begin{array}{l}12 \\
\rrbracket 30.0 \% \bigotimes\end{array}$ \\
\hline 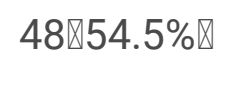 & $\begin{array}{l}22 \\
\square 45.8 \% \rrbracket\end{array}$ & $\begin{array}{l}16 \\
₫ 33.3 \% \rrbracket\end{array}$ & 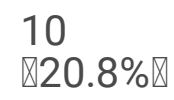 \\
\hline
\end{tabular}

0.981

0.612

$51 \rrbracket 58.0 \% \bigotimes \quad 22$

$\triangle 43.1 \% \bigotimes$

17

\33.3\%区

12

$₫ 23.5 \% \rrbracket$

0.193

0.908

$37 ه 42.0 \% \rrbracket \quad 22$

\43.2\%区

42

$\triangle 29.7 \% \rrbracket$

12

\27.0\%区

\section{Position}

Colon

$57 \rrbracket 64.8 \% \rrbracket \quad 21$

\36.8\%》

20

\35.1\%区

16

$\varangle 28.1 \% \rrbracket$

4.218

0.377

Rectum

$27 \llbracket 30.7 \% \rrbracket$

\section{5}

$\triangle 55.6 \% \rrbracket$

6

$\llbracket 22.2 \% \rrbracket$

6

Colorectum

$4 \llbracket 4.5 \% \rrbracket$

$2 \llbracket 50.0 \% \rrbracket$

2

$\triangle 50.0 \% \rrbracket$

\section{Tumor sizelmaximum}

diameter[

\begin{tabular}{|c|c|c|c|c|c|}
\hline$<5 \mathrm{~cm}$ & $46 \rrbracket 52.3 \% \rrbracket$ & $\begin{array}{l}17 \\
\text { 『37.0\%区 }\end{array}$ & 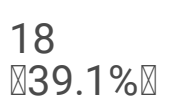 & 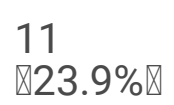 & 2.530 \\
\hline$>=5 \mathrm{~cm}$ & $42 \rrbracket 47.7 \% \rrbracket$ & $\begin{array}{l}21 \\
\square 50.0 \% \bigotimes\end{array}$ & $\begin{array}{l}10 \\
\square 23.8 \% \rrbracket\end{array}$ & $\begin{array}{l}11 \\
₫ 26.2 \% \bigotimes\end{array}$ & \\
\hline
\end{tabular}

\section{Histology}

Poor

13囚14.8\%】 $\quad 6 \rrbracket 46.2 \% \rrbracket$

2

\15.4\%区 $₫ 38.5 \% \bigotimes$

5.948

0.203

Moderate

$65 \rrbracket 73.9 \% \rrbracket$

25

\38.5\%区

24

16

Well

10冈11.4\%区 7ه70.0\%区

2

邓20.0\%】 \10.0\%区

\section{T stage}

Tis

$181.1 \% \bigotimes$

$\stackrel{1}{\otimes 100.0 \% \bigotimes}$

$0 \otimes 0.0 \% \bigotimes \quad 0 \otimes 0.0 \% \rrbracket$

19.325

0.013 
$\mathrm{T} 1$

T2

T3

T4

\section{Lymphatic metastasis}

Negative

Positive

\section{Tumor deposits}

Absent

Present

\section{Mucinous component}

Absent

Present

\section{Distant metastasis}

Negative

Positive

Microstatellite instability

MSS

MSI-L

MSI-H
$3 \llbracket 3.4 \% \rrbracket$

$12 \otimes 13.6 \% \rrbracket$

$57 \rrbracket 64.8 \% \rrbracket$

$15 \bigotimes 17.0 \% \rrbracket$
3
$\otimes 100.0 \% \bigotimes$

10

\83.3\%区

21

$\llbracket 36.8 \% \square$

$3 \rrbracket 20.0 \% \rrbracket$

$0 \rrbracket 0.0 \% \rrbracket \quad 0 \rrbracket 0.0 \% \rrbracket$

\section{2}

\16.7\%区

$0 \rrbracket 0.0 \% \rrbracket$

\section{1}

\36.8\%区

15

\26.3\%区

5

$\triangle 33.3 \% \bigotimes \quad \square 46.7 \% \square$

\begin{tabular}{|c|c|c|c|c|c|}
\hline $48 \rrbracket 54.5 \% \rrbracket$ & $\begin{array}{l}28 \\
\rrbracket 58.3 \% 区\end{array}$ & 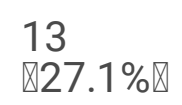 & $\begin{array}{l}7 \\
\text { Q14.6\%区 }\end{array}$ & 10.941 & 0.004 \\
\hline$\$ 4$ & $\begin{array}{l}10 \\
\rrbracket 25.0 \% \rrbracket\end{array}$ & $\begin{array}{l}15 \\
\varangle 37.5 \% \rrbracket\end{array}$ & 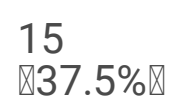 & & \\
\hline
\end{tabular}

$75 \llbracket 85.2 \% \rrbracket$

36

24

$\varangle 48.0 \% \square$

$\varangle 32.0 \% \rrbracket$

15

$\rrbracket 20.0 \% \rrbracket$

$\begin{array}{ll}7.812 & 0.020\end{array}$

$13 \otimes 14.8 \% \rrbracket \quad 2 \bigotimes 15.4 \% \rrbracket$

4

$\rrbracket 30.8 \% \rrbracket$

7

$\varangle 53.8 \% \rrbracket$ 
Positive

$1 \otimes 1.1 \% \bigotimes \quad 0 \otimes 44.4 \% \bigotimes$

0

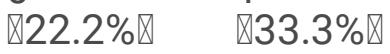

\section{TNM stage}

\begin{tabular}{|c|c|c|c|c|c|c|}
\hline 0 & $2 \rrbracket 2.3 \% \rrbracket$ & $2(100.0 \%)$ & $0 \otimes 0.0 \% \bigotimes$ & $0 \rrbracket 0.0 \% \rrbracket$ & 20.396 & 0.009 \\
\hline I & $10 \otimes 11.4 \% \bigotimes$ & $9 \varangle 90.0 \% \rrbracket$ & $\begin{array}{l}1 \\
\otimes 10.0 \% \bigotimes\end{array}$ & $0 \rrbracket 0.0 \% \rrbracket$ & & \\
\hline II & $31 \rrbracket 35.2 \% \bigotimes$ & $\begin{array}{l}16 \\
\otimes 51.6 \% \bigotimes\end{array}$ & 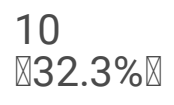 & $\begin{array}{l}5 \\
\otimes 16.1 \% \bigotimes\end{array}$ & & \\
\hline III & $42 \rrbracket 47.7 \% \bigotimes$ & $\begin{array}{l}10 \\
₫ 23.8 \% \rrbracket\end{array}$ & $\begin{array}{l}16 \\
\rrbracket 38.1 \% \rrbracket\end{array}$ & $\begin{array}{l}16 \\
\rrbracket 38.1 \% \bigotimes\end{array}$ & & \\
\hline IV & $3 \llbracket 3.4 \% \rrbracket$ & 1ه33.3\%》 & $\begin{array}{l}1 \\
\rrbracket 33.3 \% \rrbracket\end{array}$ & 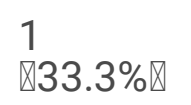 & & \\
\hline
\end{tabular}

\section{Figures}

\section{Figure 1}

CRC tumor cells can recruit fibroblasts around tumor buddings. (a) Representative H\&E images of CRC tissue in the invasive front without (the upper panel) or with (the lower panel) tumor buddings. The red arrows indicate the direction of tumor invasion and the red triangles indicate the tumor buddings. Data, $\mathrm{n}=209$. Scale bar, $100 \mu \mathrm{m}$. (b) Representative a-SMA, CD90, FAP IHC staining images of CRC tissue in the invasive front without (the upper panel) or with (the lower panel) tumor buddings. The red arrows indicate the direction of tumor invasion and the red triangles indicate the tumor buddings. Data, $n=17$. Scale bar, $100 \mu \mathrm{m}$. (c) Representative E-cad, VIM and a-SMA immunofluorescence images of normal colorectal fibroblast cell line (CCD-18Co) and primary normal colorectal fibroblast (NF1). CRC tumor cell line SW620 is the positive control for E-cad and the negative control for VIM and a-SMA. Scale bar, 20 $\mu \mathrm{m}$. (d) Model diagram of recruitment assay of CRC tumor cells to fibroblasts. (e) Recruitment assay showing the recruitment ability of medium (Med), normal colorectal epithelia cell line (FHC) and $8 \mathrm{CRC}$ tumor cell lines (LS174T, RKO, DLD-1, Caco2, SW620, HCT-8, HCT116, HCT-15) to CCD-18Co or primary normal colorectal fibroblast (NF1). Scale bar, $100 \mu \mathrm{m}$. Quantification of cell numbers of recruited fibroblasts are shown in the upper panel. Data, mean $\pm S D ; n=5$. (f) Representative morphology images of tumor formation in caecum (the upper line) and H\&E images of normal caecum and tumor formation in caecum (the lower 2 lines) in nude mice. The blue arrows indicate the tumors and the red arrows indicate the direction of tumor invasion. Data, normal, $n=5 ;$ tumor, $n=5$. Scale bar, 50 $\mu$ m. E-cad, E-cadherin; VIM, Vimentin. ns, no significance; *, $\mathrm{P}<0.05 ; * \star, \mathrm{P}<0.01 ; * \star \star, P<0.001$ by two-tailed Student's t test (e). 
A
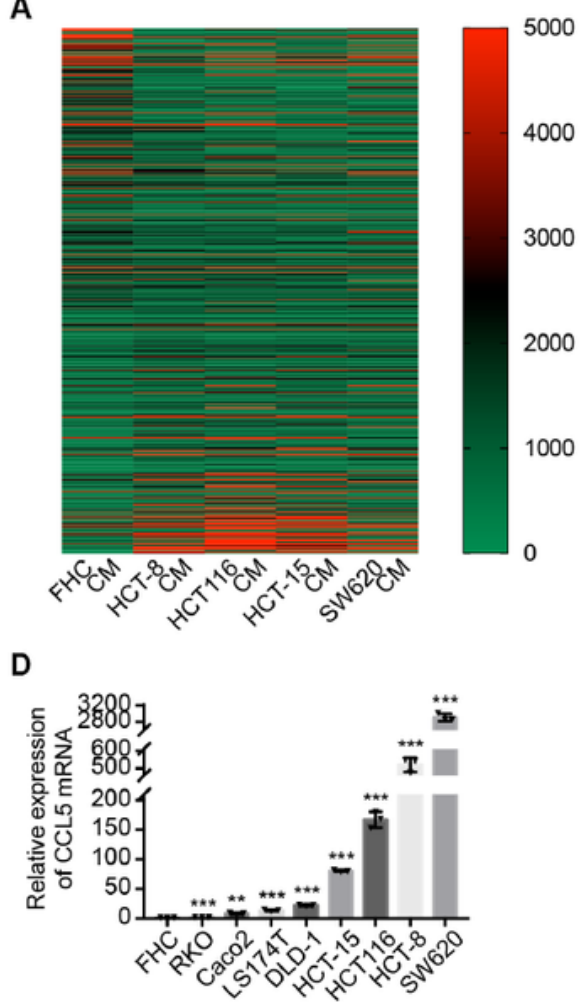

B

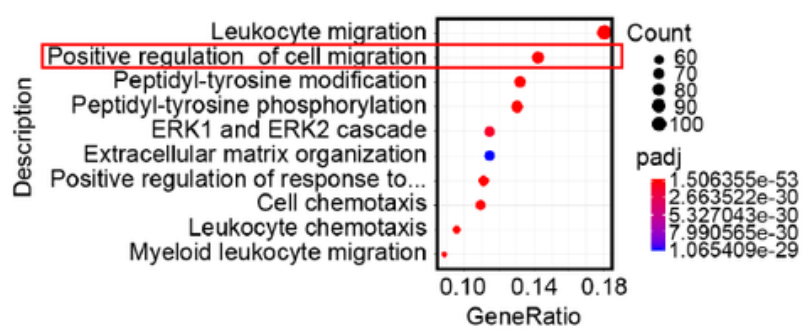

C
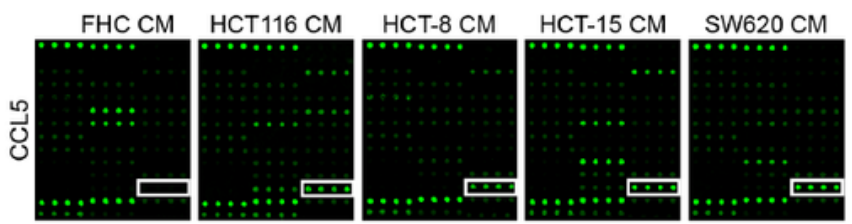
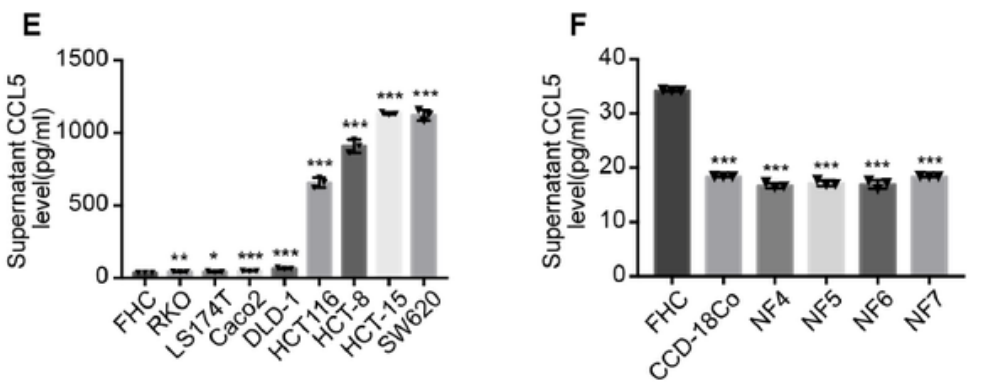

G

Tumor invasive front

H
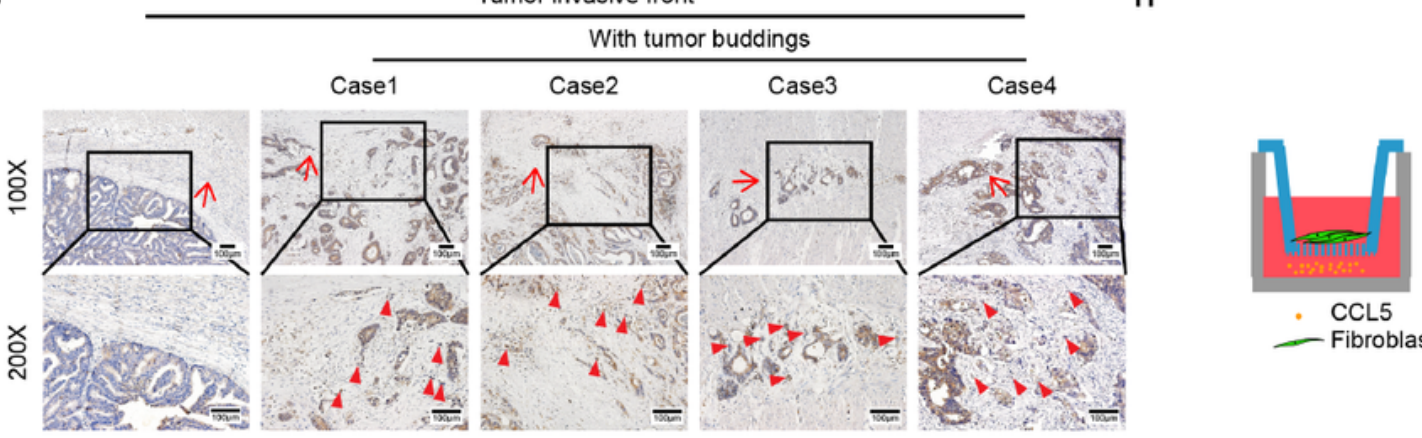

- CCL5

Fibroblast
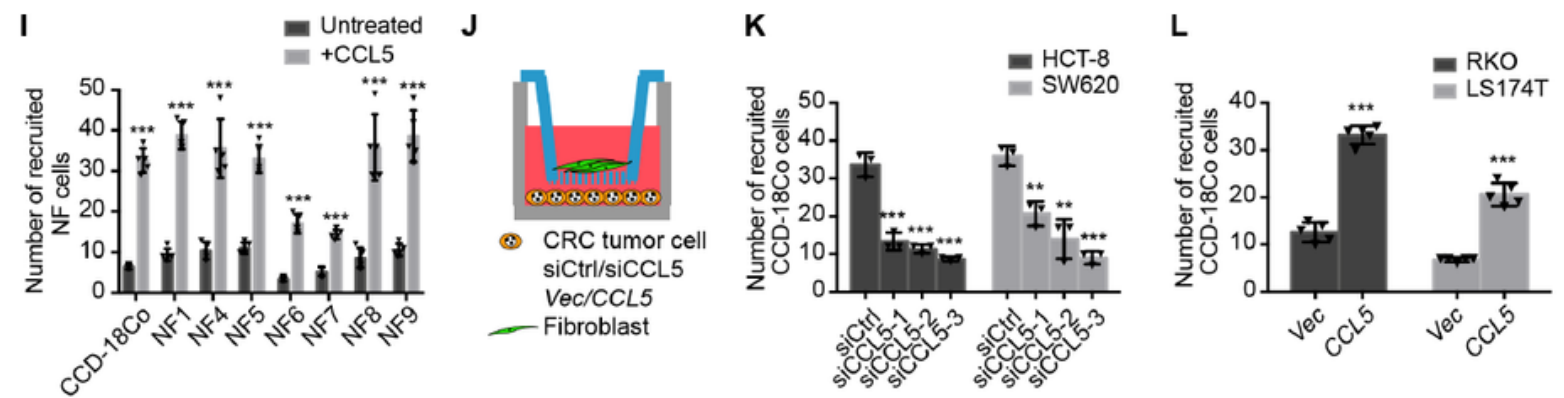

Figure 2

CRC tumor cells in tumor buddings can recruit fibroblasts through CCL5. (a) Heatmap of differential proteins in the cytokine array of the $\mathrm{CMs}$ of $\mathrm{FHC}$ and $4 \mathrm{CRC}$ tumor cell lines owning strong recruitment ability (HCT-8, HCT116, HCT-15, SW620). The cytokine array detected 1000 cytokine. (b) Differential proteins in the cytokine were analyzed by GO biological process function enrichment analysis. (c) Part of the cytokine array including CCL5 showing the expression of CCL5 in the CMs of FHC and HCT116, HCT- 
8, HCT-15, SW620. (d) Relative expression of CCL5 mRNA in FHC and 8 CRC tumor cell lines. Data, mean $\pm S D ; n=3$. (e) Supernatant CCL 5 levels in the CMs of FHC and 8 CRC tumor cell lines. Data, mean \pm SD; $n=3$. (f) Supernatant CCL5 levels in the CMs of FHC, CCD-18Co and primary normal colorectal fibroblasts (NF4, NF5, NF6, NF7). Data, mean \pm SD; $n=3$. (g) Representative CCL5 IHC staining images of CRC tissue in the invasive front without (the left column) or with (the right 4 columns) tumor buddings. The red arrows indicate the direction of tumor invasion and the red triangles indicate the tumor buddings. Scale bar, $100 \mu \mathrm{m}$. (h) Model diagram of recruitment assay of CCL5 to fibroblasts. (i) Quantification of cell numbers of CCD-18Co and primary normal colorectal fibroblasts (NF1, NF4, NF5, NF6, NF7, NF8, NF9) recruited by $40 \mathrm{ng} / \mathrm{ml}$ CCL5. Data, mean $\pm S D ; n=5$. (j) Model diagram of recruitment assay of CRC tumor cells in which CCL5 was downregulated or upregulated to fibroblasts. (k) Quantification of cell numbers of CCD-18Co recruited by HCT-8/siCtrl, HCT-8/siCCL5 and SW620/siCtrl, SW620/siCCL5. Data, mean \pm SD; $n=5$. (I) Quantification of cell numbers of CCD-18Co recruited by RKO/Vec, RKO/CCL5 and LS174T/Vec, LS174T/CCL5. Data, mean \pm SD; $n=5$. ns, no significance; * $\mathrm{P}<0.05$; **, $\mathrm{P}<0.01$; $* \star \star, P<$ 0.001 by two-tailed Student's t test (d, e, f, i, k, l). 
A

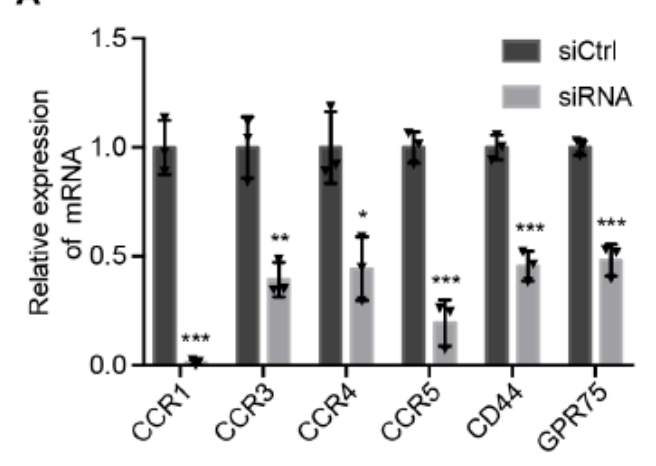

C

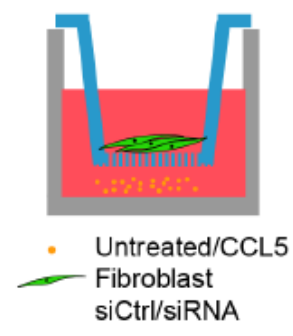

B

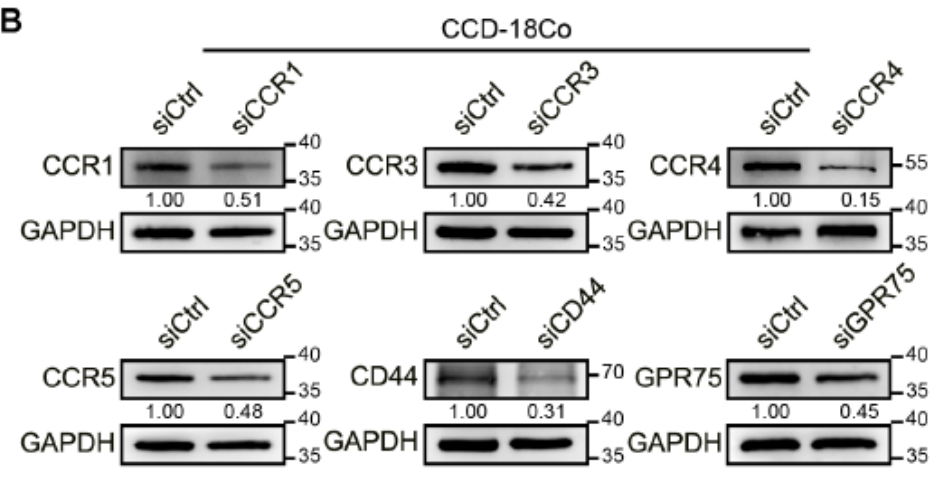

D

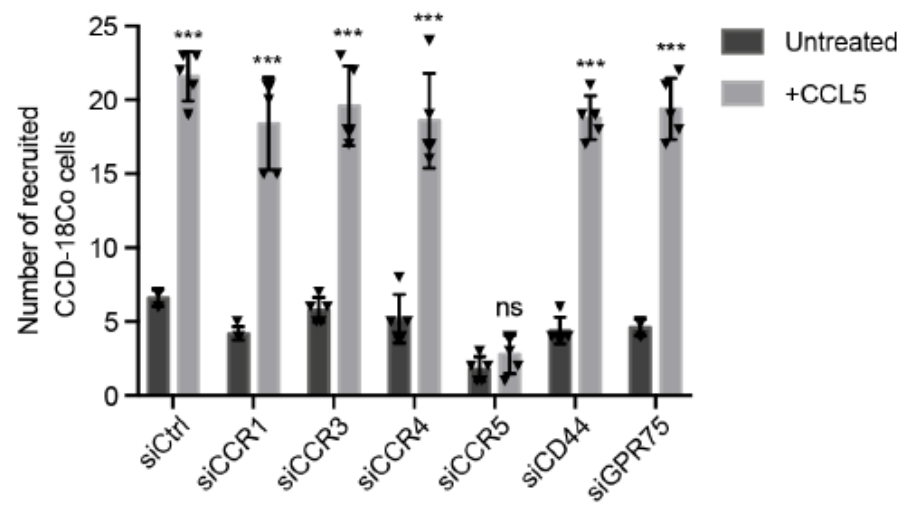

CCD-18Co

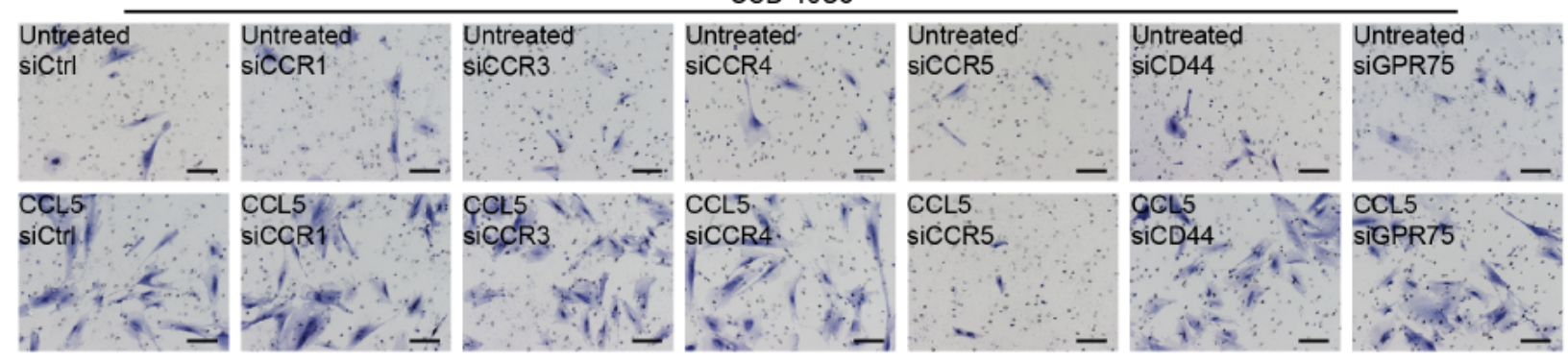

E

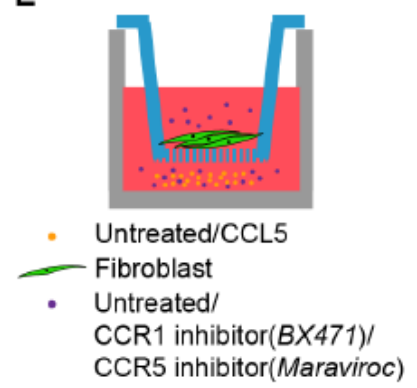

$\mathbf{F}$

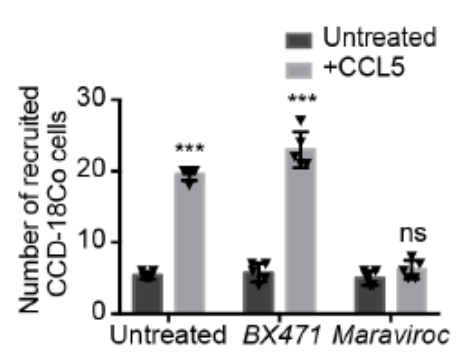

CCD-18Co

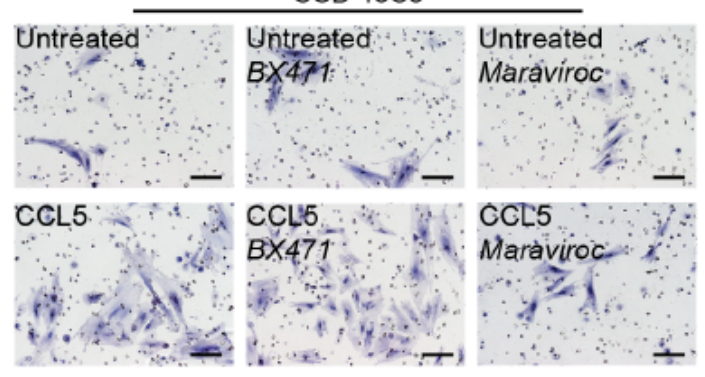

Figure 3

CCL5 recruits fibroblasts through CCR5 receptor. (a) Relative expression of CCR1, CCR3, CCR4, CCR5, CD44, GPR75 mRNA in CCD-18Co/siCtrl and CCD-18Co/siRNA. Data, mean \pm SD; $n=3$. (b) Immunoblots for CCR1, CCR3, CCR4, CCR5, CD44, GPR75 protein expression in CCD-18Co/siCtrl and CCD-18Co/siRNA. (c) Model diagram of recruitment assay of $40 \mathrm{ng} / \mathrm{ml} \mathrm{CCL5}$ to fibroblasts in which CCR1, CCR3, CCR4, CCR5, CD44 or GPR75 was downregulated. (d) Recruitment assay showing the recruitment ability of 
40ng/ml CCL5 to CCD-18Co/siCtrl and CCD-18Co/siRNA. Scale bar, 100 $\mu \mathrm{m}$. Quantification of cell numbers of recruited CCD-18Co cells are shown in the upper panel. Data, mean $\pm S D ; n=5$. (e) Model diagram of recruitment assay of $40 \mathrm{ng} / \mathrm{ml} \mathrm{CCL} 5$ to fibroblasts without or with CCR1 inhibitor (BX471) or CCR5 inhibitor (Maraviroc). (f) Recruitment assay showing the recruitment ability of $40 \mathrm{ng} / \mathrm{ml}$ CCL5 to

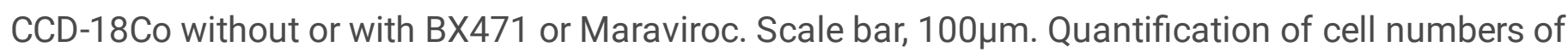
recruited CCD-18Co cells are shown in the left panel. Data, mean $\pm S D ; n=5$. ns, no significance; *, $P<$ $0.05 ; * \star, P<0.01 ; * \star \star, P<0.001$ by two-tailed Student's t test $(a, d, f)$.

A

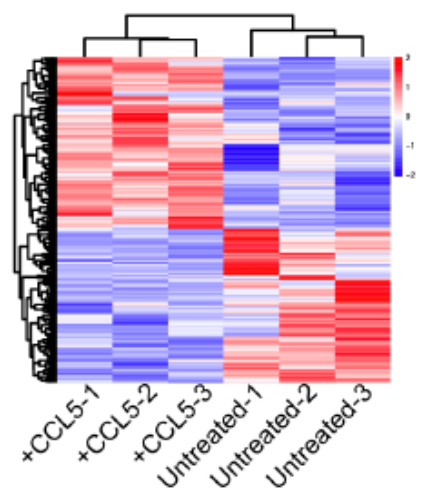

D

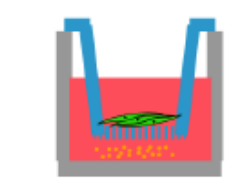

Untreated/CCL5

_ Fibroblast

siCtrl/siSLC25A24
B
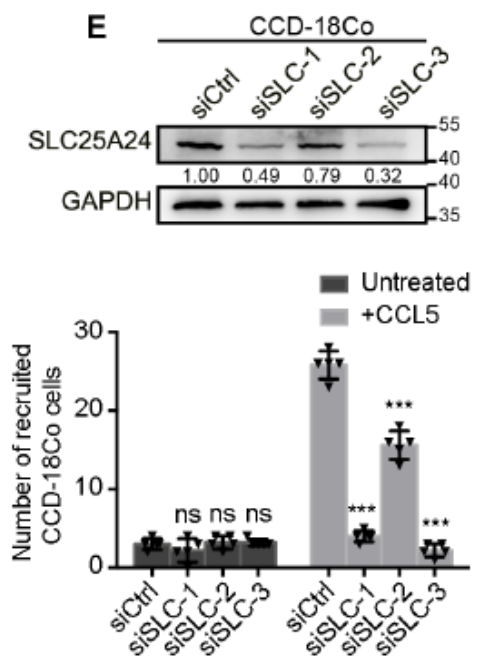

H

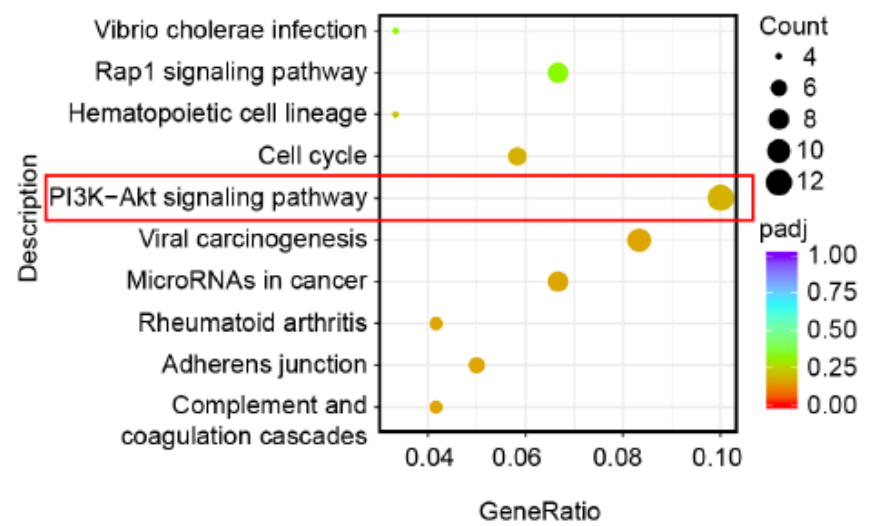

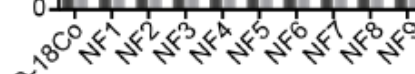
$\mathrm{CO}^{\circ}$
C

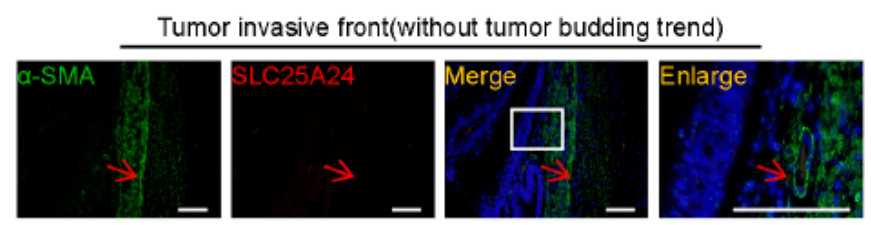

Tumor invasive front(with tumor budding trend)

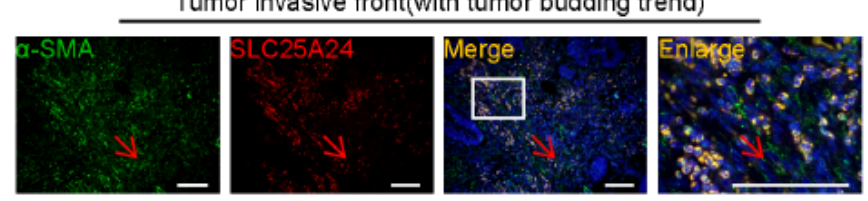

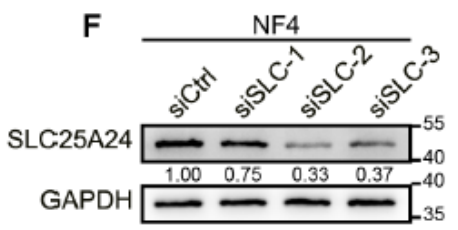
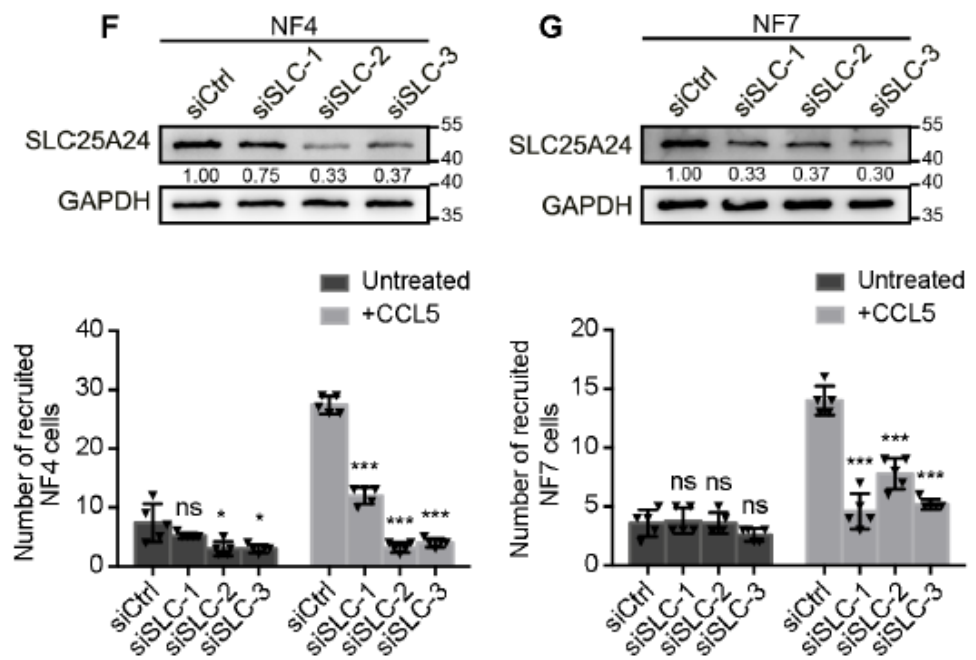

I

CCD-18Co

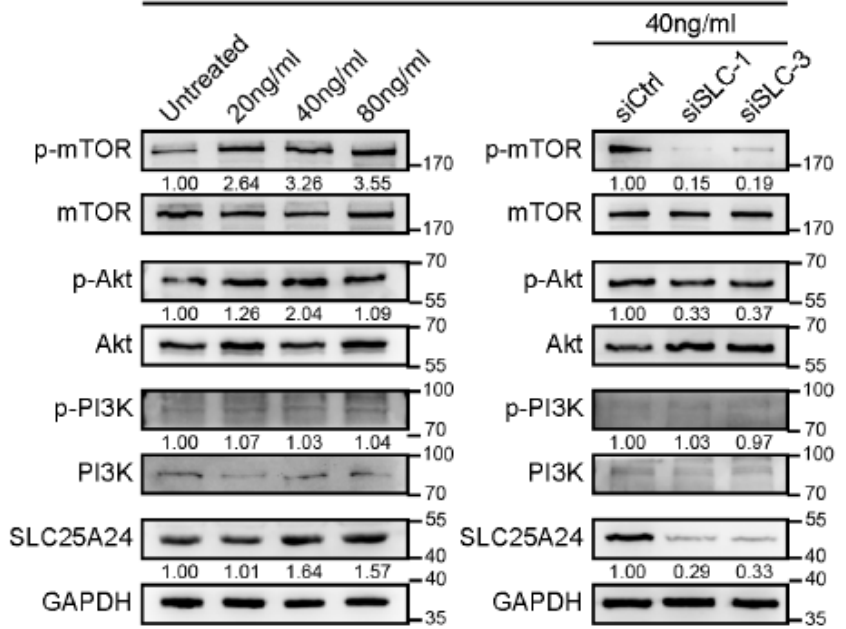




\section{Figure 4}

CCL5 recruits fibroblasts through SLC25A24 inside fibroblasts. (a) Heatmap of differential genes in the transcriptome sequencing of CCD-18Co before and after 40ng/ml CCL5 stimulation. Data, $\mathrm{n}=3$. (b) Relative expression of SLC25A24 mRNA in CCD-18Co and primary normal colorectal fibroblasts (NF1, NF2, NF3, NF4, NF5, NF6, NF7, NF8, NF9) before and after 40ng/ml CCL5 stimulation. Data, mean \pm SD; $\mathrm{n}=3$. (c) Representative a-SMA and SLC25A24 immunofluorescence images in the invasive front of CRC tissue without (the upper panel) or with (the lower panel) tumor budding trend. The red arrows indicate the direction of tumor invasion. Data, $n=10$. Scale bar, $100 \mu \mathrm{m}$. (d) Model diagram of recruitment assay of $40 \mathrm{ng} / \mathrm{ml}$ CCL5 to fibroblasts in which SLC25A24 was downregulated. Immunoblots for SLC25A24 protein expression and quantification of cell numbers recruited by $40 \mathrm{ng} / \mathrm{ml}$ CCL 5 of CCD-18Co/siCtrl and CCD18Co/siSLC25A24 (e), NF4/siCtrl and NF4/siSLC25A24 (f), NF7/siCtrl and NF7/siSLC25A24 (g). Data, mean $\pm S D ; n=5$. (h) Differential genes in the transcriptome sequencing were analyzed by KEGG pathway enrichment analysis. (i) Immunoblots for SLC25A24, PI3K, p-PI3K, Akt, p-Akt, mTOR, p-mTOR protein expression in CCD-18Co before and after different concentration of CCL5 stimulation (the left panel) and those proteins in CCD-18Co/siCtrl and CCD-18Co/siSLC25A24 with 40ng/ml CCL5 stimulation (the right panel). SLC, SLC25A24; p-PI3K, phosphorylated PI3K; p-Akt, phosphorylated Akt; p-mTOR, phosphorylated mTOR. ns, no significance; ${ }^{*} \mathrm{P}<0.05 ; \star \star, \mathrm{P}<0.01$; ${ }^{\star \star \star}, \mathrm{P}<0.001$ by two-tailed Student's $t$ test $(\mathrm{b}, \mathrm{e}, \mathrm{f}, \mathrm{g})$.

\section{Figure 5}

CCL5 contributes to the increase of a-SMA+CD90+FAP- fibroblasts to promote tumor angiogenesis. (a) Representative a-SMA, FAP and CD90 immunofluorescence images of CCD-18Co and primary normal colorectal fibroblast (NF1) before and after $40 \mathrm{ng} / \mathrm{ml} \mathrm{CCL} 5$ stimulation. Scale bar, $40 \mu \mathrm{m}$. (b) Immunoblots for a-SMA, CD90, FLI1, VE-cad, CD31 and VEGFA protein expression in CCD-18Co and primary normal colorectal fibroblast (NF1) before and after 40ng/ml CCL5 stimulation. (c) Matrigel angiogenesis experiment showing the angiogenesis ability of CCD-18Co and primary normal colorectal fibroblasts (NF1, NF2, NF3) before and after 40ng/ml CCL5 stimulation. Scale bar, 100 $\mu$ m. Quantification of increasing folds of tube formation are shown in the left panel. Data, mean $\pm S D ; n=3$. (d) Representative CCL5, a-SMA, CD90, FAP and CD31 IHC staining images of CRC tissue in the invasive front without (the upper panel) or with (the lower panel) tumor buddings. The red arrows indicate the direction of tumor invasion and the red triangles indicate the tumor buddings. Data, $n=17$. Scale bar, $100 \mu \mathrm{m}$. VE-cad, VEcadherin. ns, no significance; ${ }^{*}, \mathrm{P}<0.05 ;{ }^{* \star}, \mathrm{P}<0.01 ; * \star *, \mathrm{P}<0.001$ by two-tailed Student's t test (c). 
A

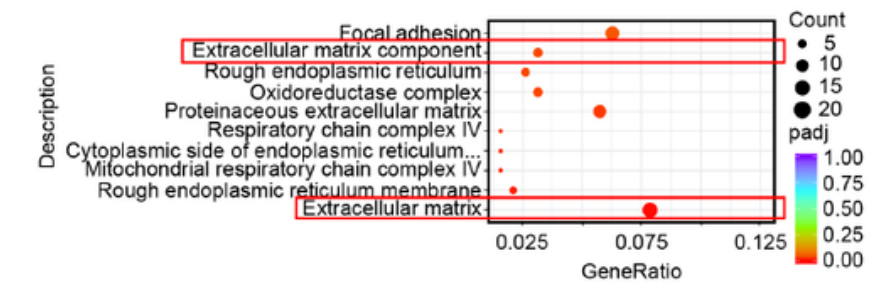

C

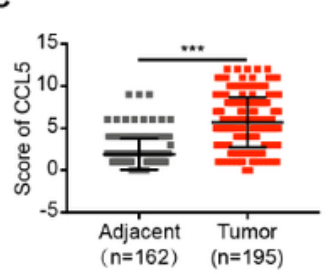

D

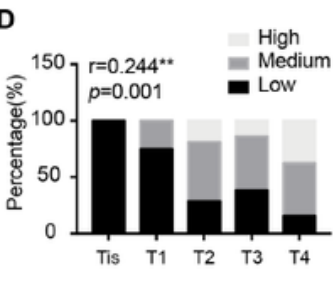

B

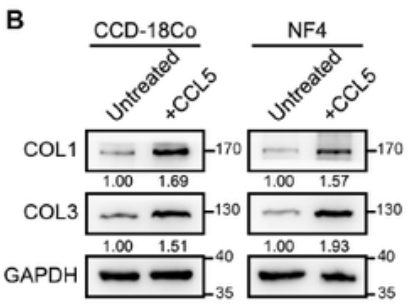

E
F

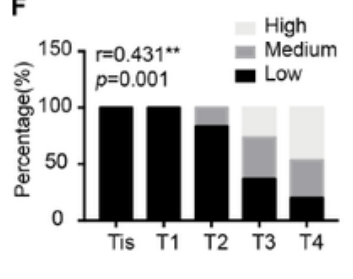

G
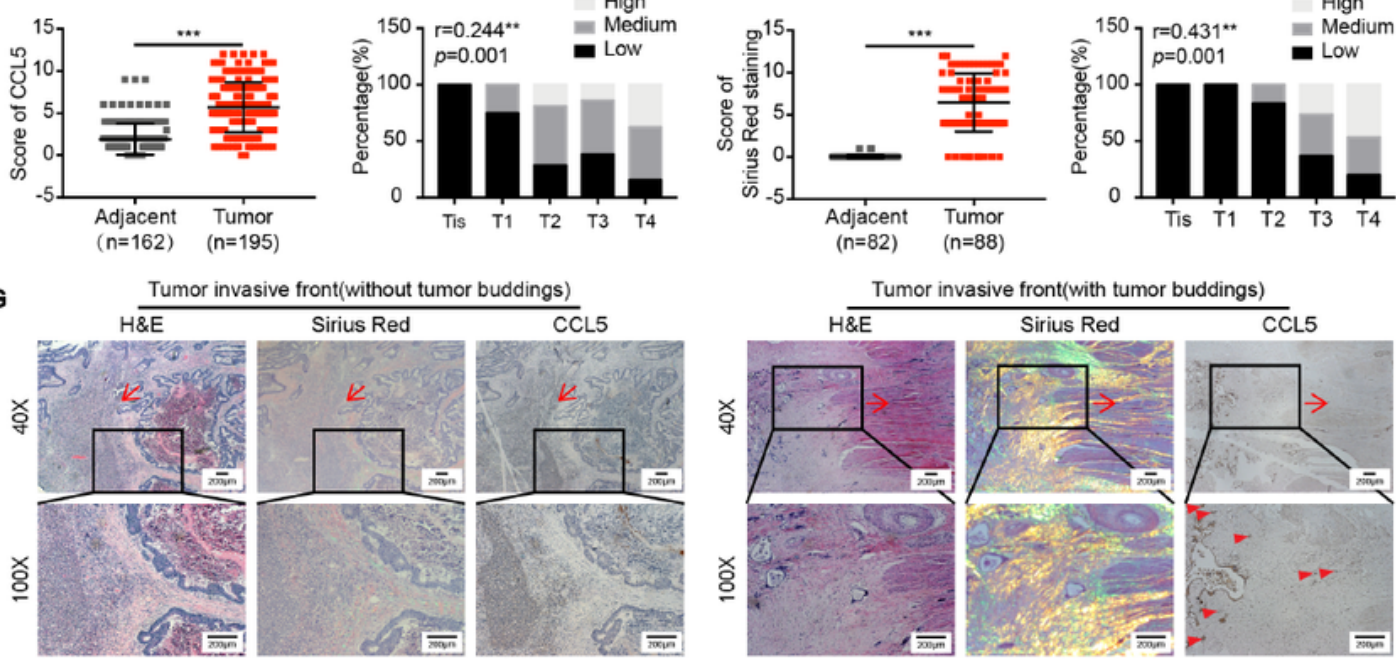

H

I

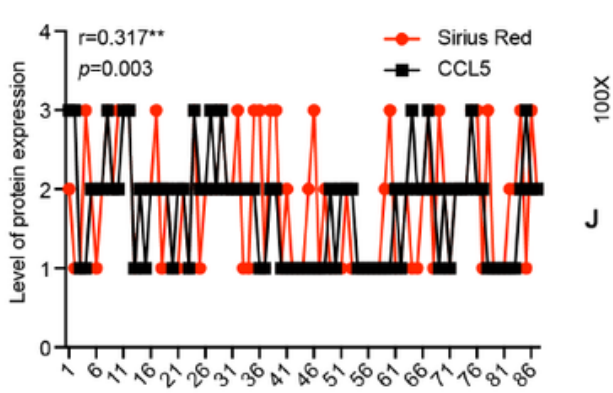

K
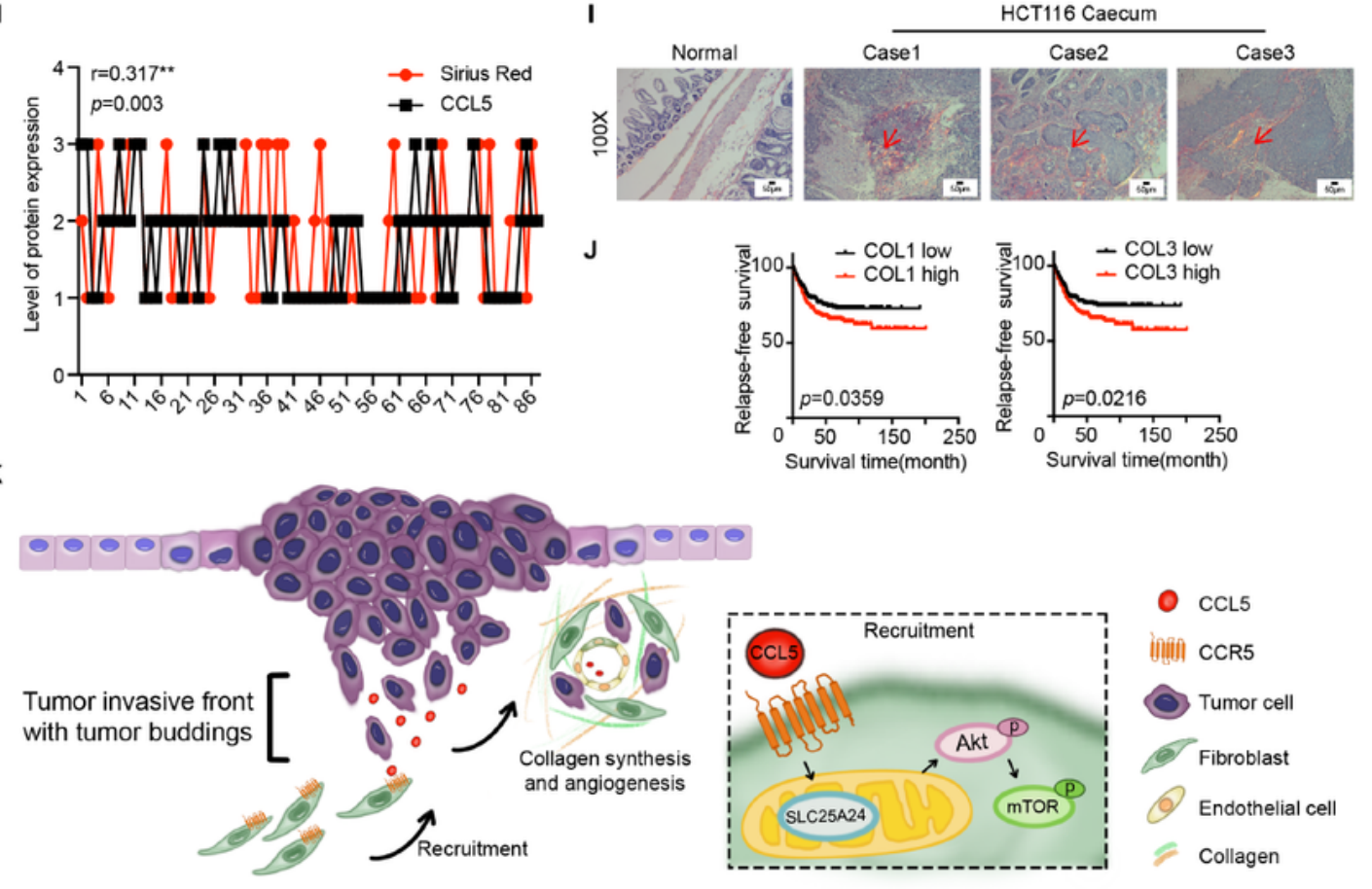

- CCL5

infiif CCR5

( $)$ Tumor cell

Fibroblast

- Endothelial cell

collagen

\section{Figure 6}

CCL5 promotes collagen synthesis through fibroblasts contributing to tumor progression. (a) Differential genes in the transcriptome sequencing were analyzed by GO function enrichment analysis. (b) Immunoblots for COL1 and COL3 protein expression in CCD-18Co and primary normal colorectal fibroblast (NF4) before and after 40ng/ml CCL5 stimulation. (c) IHC score of CCL5 in adjacent normal tissues and CRC tumour tissues. Data, adjacent, $n=162$; tumor, $n=195$. (d) Spearman's correlation analysis 
of high and low expression of CCL5 in CRC patients with different depth of tumor invasion. Spearman $r=$ 0.244. Data, $n=195$. (e) Score of Sirius Red staining in adjacent normal tissues and CRC tumour tissues. Data, adjacent, $n=82$; tumor, $n=88$. ( $f$ ) Spearman's correlation analysis of high and low staining of Sirius Red in CRC patients with different depth of tumor invasion. Spearman $r=0.431$. Data, $n=88$. $(\mathrm{g})$ Representative CCL5 IHC staining and Sirius Red staining images of CRC tissue in the invasive front without (the left panel) or with (the right panel) tumor buddings. The red arrows indicate the direction of tumor invasion and the red triangles indicate the tumor buddings. Scale bar, 200 $\mu \mathrm{m}$. (h) Spearman's correlation analysis between CCL5 expression and Sirius Red staining in CRC tissues. Spearman $r=$ 0.317. Data, $n=88$. (i) Representative Sirius Red staining images of normal caecum and tumor formation in caecum in nude mice. Data, normal, $n=5$; tumor, $n=5$. Scale bar, $50 \mu m$. (j) Kaplan-Meier survival analysis of CRC patients with low and high expression of COL1 and COL3 in the CRC microarray profiles GSE39582. Data, $n=566$. ( $k$ ) Schematic diagram of the contribution of tumor budding derived-CCL5 to

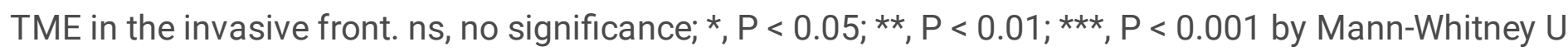
test (c, e), Spearman's correlation test $(d, f, h)$, log-rank test $(j)$.

\section{Supplementary Files}

This is a list of supplementary files associated with this preprint. Click to download.

- Additionalfile1FigureS1.pdf

- Additionalfile2Figures2.pdf

- Additionalfile3FigureS3.pdf

- Additionalfile4FigureS4.pdf

- Additionalfile5TableS1.xlsx

- Additionalfile6Tables2.xlsx

- Additionalfile7TableS3.xIsx 